Review Article

\title{
The Outcome of Hydroxychloroquine in Patients Treated for COVID-19: Systematic Review and Meta-Analysis
}

\author{
Teshale Ayele Mega $\mathbb{D}^{1},{ }^{1}$ Temesgen Mulugeta Feyissa, ${ }^{2}$ Dula Dessalegn Bosho, ${ }^{2}$ \\ Kabaye Kumela Goro, ${ }^{2}$ and Getandale Zeleke Negera $\mathbb{D}^{2}$ \\ ${ }^{1}$ Deaprtment of Pharmacology and Clinical Pharmacy, School of Pharmacy, College of Health Sciences, Addis Ababa University, \\ Addis Ababa, Ethiopia \\ ${ }^{2}$ Department of Clinical Pharmacy, School of Pharmacy, Institute of Health, Jimma University, Jimma, Ethiopia
}

Correspondence should be addressed to Teshale Ayele Mega; teshale@mtu.edu.et

Received 3 June 2020; Revised 12 August 2020; Accepted 18 September 2020; Published 13 October 2020

Academic Editor: Hisao Imai

Copyright ( $\odot 2020$ Teshale Ayele Mega et al. This is an open access article distributed under the Creative Commons Attribution License, which permits unrestricted use, distribution, and reproduction in any medium, provided the original work is properly cited.

Background. The pandemic of coronavirus disease 2019 (COVID-19) caused by severe acute respiratory syndrome coronavirus 2 (SARS-CoV-2) resulted in an unprecedented public health challenge worldwide. Despite urgent and extensive global efforts, the existing evidence is inconclusive regarding the medications used for the treatment of COVID-19. Purpose. To generate an up-todate evidence for the clinical safety and efficacy of hydroxychloroquine (HCQ) with or without azithromycin (AZ) among patients treated for COVID-19. Data Source. PubMed, Cochrane CENTRAL, LITCOVID, Web of Science, SCOPUS, BioRxiv, Embase, MedRxiv, and Wiley online library were searched from 2019/12/30 to 2020/05/23. Study Selection. Three investigators assessed the quality of the studies. Data Extraction. Data about study characteristics, effect estimates, and the quality of the studies were extracted by two independent reviewers and cross-checked by the third reviewer. Data Synthesis. The data of 6,782 (HCQ group, 3623; HCQ + AZ group, 1,020; control group, 2139) participants were included. HCQ was compared with standard care for virologic efficacy, disease progression, mortality, and adverse effects. HCQ was also compared with HCQ + AZ for QTc prolongation, admission to the intensive care unit, and mortality. The study found HCQ did not alter the rate of virologic cure $(\mathrm{OR}=0.78 ; 95 \% \mathrm{CI}: 0.39-1.56)$ and the risk of mortality $(\mathrm{OR}=1.26 ; 95 \% \mathrm{CI}: 0.66-2.39)$. The pooled prevalence for mortality was $5.8 \%$ (95\% CI: 0.9\%-10.8\%). Moreover, HCQ did not impact disease progression (OR=0.9; 95\% CI: 0.36-2.29) but resulted in a higher risk of adverse effects ( $\mathrm{OR}=2.35 ; 95 \% \mathrm{CI}$ : 1.15-4.8). HCQ was also compared against HCQ + AZ, and no difference was observed in QTc prolongation above $500 \mathrm{~ms}(\mathrm{OR}=1.11 ; 95 \% \mathrm{CI}: 0.54-2.28)$, admission to the intensive care unit $(\mathrm{OR}=0.92 ; 95 \%$ CI: $0.52-1.63)$, and mortality ( $\mathrm{OR}=0.88$; 95\% CI: 0.55-1.43). However, in the analysis of single-arm studies, about $11.2 \%$ (95\% CI: 7.0\%-15.5\%) of patients have developed an absolute increase of QTc greater than $500 \mathrm{~ms}$, and $4.1 \%$ (95\% CI: $1.1 \%-7.1 \%)$ of patients discontinued their medication. Conclusion. This meta-analysis and systematic review, which included a limited number of poorly designed studies of patients with COVID-19, revealed HCQ is intolerable, unsafe, and not efficacious. Similarly, HCQ + AZ combination was not different from HCQ alone in curbing mortality and ICU admission.

\section{Introduction}

The pandemic of coronavirus disease 2019 (COVID-19) caused by severe acute respiratory syndrome coronavirus 2 (SARS-CoV-2) resulted in an unprecedented public health challenge worldwide [1]. As of May 26, 2020, there were more than 5.3 million documented cases, and over 300,000 patients have succumbed to this disease globally [2]. The morbidity and mortality due to COVID-19 have found to increase with age and the presence of comorbid conditions such as diabetes, hypertension, coronary heart disease, or chronic obstructive lung disease [3].

With the rising death toll and a vaccine unlikely very soon, extensive global efforts are underway to develop safe and effective therapeutics against COVID-19 [4]. Among the efforts undergoing to treat the disease, repurposing of old medications is a compelling strategy for which their safety 
profile, pharmacokinetics, and potential drug interactions are well studied [5].

Initially, a combination of lopinavir and ritonavir was utilized as the first-line agent in Wuhan, China, the epicenter of the disease. However, a previous study [5] failed to show the beneficial clinical effects of this combination. Indeed, it has received considerable criticism from the scientific community [6].

Meanwhile, the aminoquinolines, chloroquine (CQ), and hydroxychloroquine (HCQ) have emerged as a potent inhibitor of SARS-CoV-2 in vitro, and some studies also demonstrated their clinical benefit among hospitalized COVID-19 patients [7-9]. On March 30, 2020, the United States Food and Drug Administration (FDA) granted emergency authorization that allowed the use of these drugs in hospitalized COVID-19-pneumonia [10].

To date, regardless of limited evidence, HCQ with or without azithromycin (AZ) is widely utilized in clinical settings to treat thousands of COVID-19 patients around the world [4]. The studies supporting the use of HCQ had suffered from methodological flaws including small sample size and ill quality of design creating difficulty in measuring the true clinical effects. The first study from France showed HCQ and AZ combination as an effective therapy for COVID-19. In this open-label non-randomized clinical trial, a total of 20 patients were treated with HCQ at a dose of $200 \mathrm{mg}$ three times daily for 10 days, and the data showed a significant reduction in viral carriage at day 6 post-inclusion compared to controls $(70.0 \%$ clearance by day 6 vs. $12.5 \%$ clearance by day 6 in control groups). Interestingly, the addition of AZ to HCQ $(n=6)$ resulted in a $100 \%$ virological cure on day 6 postinclusion, compared with $57.1 \%$ virological cure in the HCQ alone arm $(n=14)$ and $12.5 \%$ virological cure in the control arm $(n=16)$ [8]. Similarly, Million et al. [11] showed HCQ/AZ combination to be safe with a lower death rate. On the contrary, a more recent study conducted by Molina and his colleagues [12] failed to show evidence of a strong antiviral activity or clinical benefit of HCQ in combination with AZ for the treatment of hospitalized patients with severe COVID-19. Notably, the patients included in this study belonged to the severe COVID-19 category and had significant comorbidities including solid and hematological cancers, HIV, and obesity.

A large observational study conducted in the USA reported that HCQ use among patients hospitalized with COVID-19 was not associated with either a greatly lowered or increased risk of intubation or death [4]. A very recent study by Mahévas et al. [13] and Rosenberg et al. [14] also did not show significant differences in terms of in-hospital mortality among patients receiving HCQ with/without AZ compared with standard care. Besides, a systematic review by Sarma and his colleagues [15] concluded that treatment with HCQ had benefits in terms of fewer cases showing radiological progression, time to body temperature normalization, and the number of cough days compared to standard treatment. However, no difference was seen in terms of virological cure, death, or clinical worsening of the disease.
The safety of HCQ with/without AZ in COVID-19 patients, including cardiac arrest and QTc prolongation, was also investigated by several studies $[14,16]$. Interestingly, both drugs can potentially cause QTc prolongation, leading to life-threatening ventricular arrhythmias and torsade de pointes [17]. Critically ill admitted COVID-19 patients with multiorgan failure and metabolic derangements and those having other drugs that can increase the risk of QTc prolongation are at greater risk [18]. A study conducted in New York recorded higher rates of cardiac arrest among patients receiving a combination of HCQ and AZ [14]. Similarly, Ramireddy et al. [19] reported a significant number of patients with QTc-interval prolongation, and the highest QTc values were recorded in those treated with a combination of HCQ and AZ.

Despite numerous studies with small sample size, the efficacy and safety of HCQ in COVID-19 patients remained unclear. Given the inconclusiveness of the existing evidence and awaiting findings from large randomized controlled clinical trials to clear the controversy, we conducted a systematic review and meta-analysis in the interim to investigate the safety and effectiveness of HCQ in the clinical setup.

\section{Objective}

The objective of this review is to synthesize an evidence for the safety, efficacy, and tolerability of HCQ with or without AZ among patients treated for COVID-19.

\section{Methods}

This review was described by the Preferred Reporting Items for Systematic Reviews and Meta-Analysis (PRISMA) framework. The studies were identified from PubMed, Cochrane CENTRAL, LITCOVID, Web of Science, SCOPUS, BioRxiv, Embase, MedRxiv, and Wiley online library. The search was conducted to include human studies published in English language from 2019/12/30 to 2020/05/23. The search terms included 2019-nCoV, 2019 novel coronavirus, COVID-19, coronavirus disease-2019, hydroxychloroquine, Plaquenil, and hydroxychloroquine sulphate. Details of the search strategy for some databases are annexed (Appendix A).

Study designs with single-group prospective/retrospective observational studies and controlled clinical trials were pooled using meta-proportion, while prospective/retrospective observational studies and controlled clinical trials comparing HCQ with or without AZ versus usual care or HCQ with HCQ plus AZ were pooled using RevMan version 5.3. Controlled clinical trials with serious risk of bias were not included in the pooled analysis, and their findings were narrated descriptively.

\section{Selection of Studies}

After removing all irrelevant articles, TAM, TMF, and GZN independently reviewed articles for data quality and methodological validity using standardized critical appraisal 
instruments obtained from https://www.joannabriggs.org/ assets/docs/jbc/...sr.../jbi-sr-protocol-template.docx. Any disagreement was handled by consulting KKG and DDB. Data extraction was handled by DD and KK using the standardized data extraction tool available at https://www.joannabriggs.org/ assets/docs/jbc/...sr.../jbi-sr-protocol-template.docx.

4.1. Inclusion and Exclusion Criteria. Studies were considered if they included patients who received HCQ alone or in combination with other specific treatment modalities for COVID-19 infection. Both controlled clinical trials (CCTs) and observational studies with and without the comparator group were considered for inclusion. Data on at least one of the following outcomes had to be available for inclusion: virologic efficacy, mortality, disease progression, adverse effects, QTc prolongation, and drug discontinuation due to adverse effects (tolerability). Studies conducted among pediatric COVID-19 patients, case reports, preclinical studies, and studies that did not report outcomes with HCQ in COVID-19 were excluded.

4.2. Risk of Bias. The risk of bias for comparative clinical trials $[8,20-22]$ was assessed using the Cochrane risk of bias tool for randomized controlled studies [23]. The study by Gautret et al. [8] was a non-randomized clinical trial and hence assessed using the ROBINS-I scale [24]. The results of the risk of bias for the studies included in the meta-analysis are found in Appendix B. The Modified Newcastle-Ottawa Quality Assessment scale [25] was used for observational studies, and the full results are presented in Appendix B.

4.3. Outcome Assessment and Statistical Analysis. The virologic efficacy, mortality, disease progression, adverse effects, QTc prolongation, and drug discontinuation due to adverse effects (tolerability) were assessed. Virologic efficacy is defined as two negative results of SARS-CoV-2 in nasopharyngeal swab using RT-PCR assay with samples obtained 24 hours apart. Disease progression is defined as the need for admission to the intensive care unit, the need for mechanical ventilation, and hospital admission of previously mild cases. Adverse effect is defined as any adverse effect (side effect) reported in a study except QTc prolongation. QTc prolongation is defined as an increase of greater than $60 \mathrm{~ms}$ from baseline, and absolute QTc increases to greater than $500 \mathrm{~ms}$.

Open Meta [Analyst] was used to analyse the proportions of mortality, QTc prolongation, and tolerability in single-arm studies $[11,12,16,26-29]$. The pooled proportion of the outcomes was reported with its $95 \%$ confidence interval (CI). RevMan 5.3 was used to estimate the risk of virologic efficacy, mortality, disease progression, and adverse effects in studies that compared HCQ with usual care or HCQ with HCQ plus AZ. The odds ratios (ORs) and 95\% CI were calculated to estimate the effect sizes. Meta-analysis using the Mantel Hazel method was conducted, and either the fixed-effect or random-effects model was applied. A fixed-effect model was used when the heterogeneity was low to moderate [23]; otherwise, the random-effects model was applied.

\section{Results}

The databases (9 databases) search produced 442 articles. After removing the duplicates and excluding 138 articles with thorough evaluation for inclusion using titles and abstracts, 56 full-text articles were assessed for eligibility. Furthermore, 30 full-text articles were subjected to critical appraisal, and 10 articles were dropped with reasons. Twenty full-text articles ( 5 controlled clinical trials with 288 patients and 15 observational studies with 6,742 patients) were included in the final analysis. Of these, 12 $[4,8,13,14,20-22,26,30-32]$ were double-arm studies (Figure 1). These studies compared hydroxychloroquine (HCQ) either with usual/standard care or HCQ with HCQ and azithromycin (AZ). The details of the studies are described in Table 1. Two of the controlled clinical trials $[20,22]$ were at the preprint stage.

Both the double-arm (Table 1) and single-arm (Table 2) studies were subjected to meta-analysis to estimate the effects of the interventions. The outcomes assessed with double-arm studies include virologic efficacy [8, 20, 21], clinical efficacy (mortality $[4,8,13,14,30,31]$ and disease progression $[4,8,13,14,22,31,32]$ ), safety (risk of adverse effects) [8, 20-22], and tolerability and QT prolongation $[14,26,34]$. Outcomes were assessed for both HCQ versus standard care and HCQ versus $\mathrm{HCQ}+\mathrm{AZ}$. The outcome assessed in single-arm studies include the proportions of mortality, QTc prolongation [11, 12, 16, 26-28, 34], and drug discontinuation [12, 26-28].

5.1. Virologic Efficacy. To estimate the risk of virologic cure, the data of 180 patients (90 HCQ and 90 non-HCQ groups) from two controlled clinical trials $[20,21]$, with moderate risk of bias, were pooled. Although Gautret et al. [8] reported improved virologic cure rate among the HCQ group as compared to the non-HCQ group (16/20 versus $2 / 16)$, the finding was not included in the pooled data due to its serious risk of bias. Hence, the pooled result indicated that the virologic cure rate of the HCQ group was not statistically different from the non-HCQ/standard care group $(\mathrm{OR}=0.78 ; 95 \% \mathrm{CI}: 0.39-1.56)$. The test for the overall effect was $Z=0.69(p=0.49) \quad\left(\mathrm{Tau}^{2}=0.00 ; \mathrm{Chi}^{2}=0.18, \mathrm{~d} f=1\right.$ $\left.(p=0.67) ; I^{2}=0 \%\right)$ (Figure 2$)$.

5.2. Mortality. The finding was generated from five observational studies $[4,13,14,30,31]$ comprising the data of 2,864 COVID-19 patients (1,311 HCQ and 1,553 non-HCQ groups). The overall result indicated that treatment with HCQ did not result in improved survival (OR $=1.26 ; 95 \%$ CI: 0.66-2.39) as compared to the routine care. The test for the overall effect, $Z=0.71(p=0.48)$. However, the interpretation of this finding might be limited by the substantial heterogeneity; heterogeneity: $\mathrm{Tau}^{2}=0.42 ; \mathrm{Chi}^{2}=24.91, \mathrm{~d} f=4$ $(p \leq 0.001)$; and $I^{2}=84 \%$ (Figure 3 ). A controlled clinical trial [8] also reported one death out of 14 HCQ-exposed 


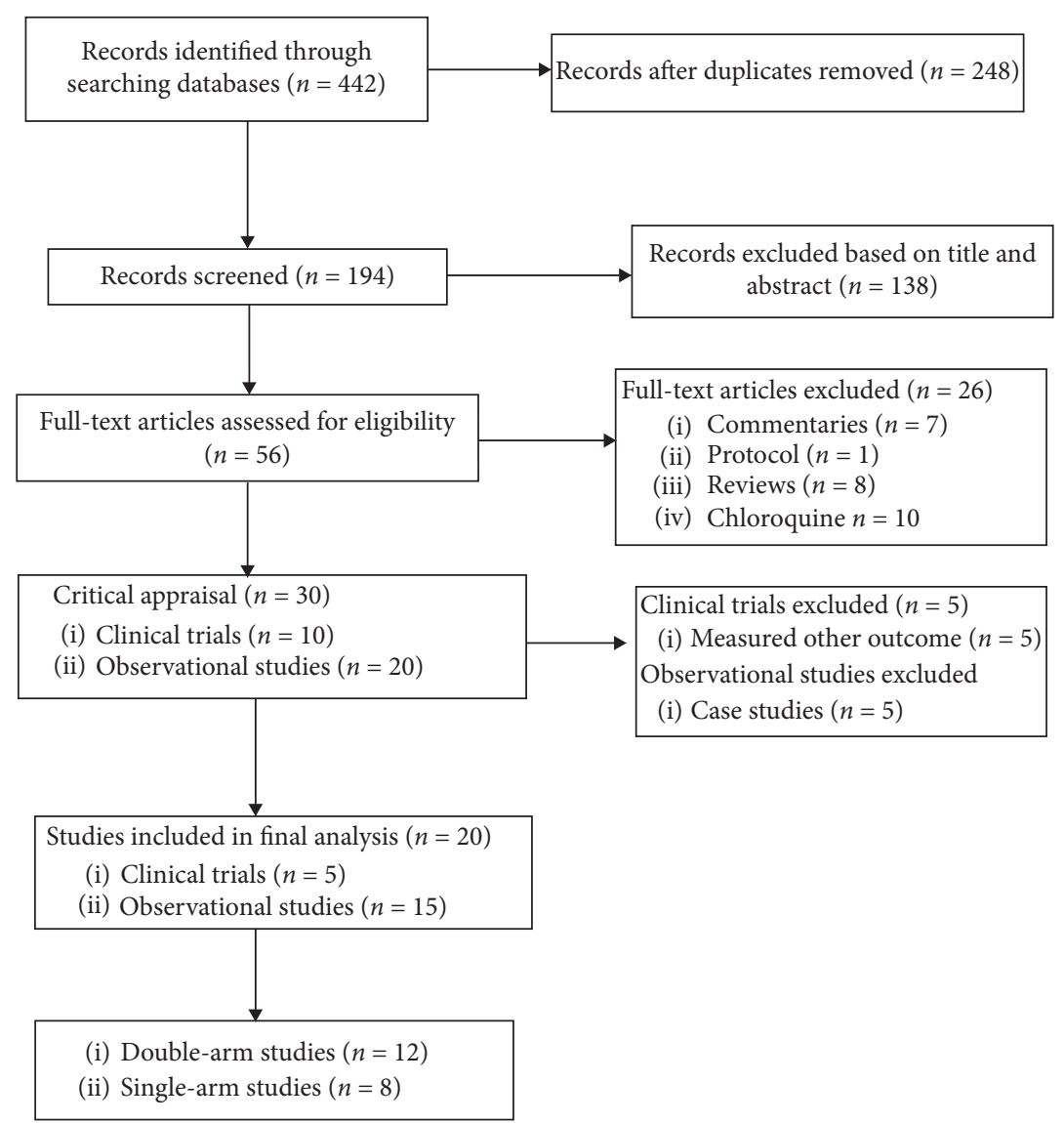

FIgURe 1: The Preferred Reporting Items for Systematic review and Meta-Analysis (PRISMA) flow chart for the included studies.

patients and no death out of 16 in the opposite arm. Of note, the study was removed from pooled analysis as it carries a serious risk of bias (Appendix A).

Likewise, the data of 1,487 COVID-19 patients from single-arm observational studies $[12,13,16,17]$ were included to determine the pooled prevalence of mortality among patients treated with HCQ with or without AZ. The pooled prevalence was 5.8\% (95\% CI: $0.9 \%-10.8 \%)$ with considerable heterogeneity $\left(I^{2}=92.28 \%, p<0.001\right)$ (Figure 4 ). The heterogeneity could be attributed to the age difference of the COVID-19 patients, as the studies with death reports had a median age of greater than 60 years.

In the three $[11,16,27]$ of the observational studies, the cause of death was respiratory and multiorgan failure. There was no death due to arrhythmogenic adverse effects.

5.3. Disease Progression. The outcome coded as "disease progression" included the need for admission to the intensive care unit, the need for mechanical ventilation, and hospital admission of previously mild cases. The data of 3,003 COVID-19 patients (1,699 HCQ and 1,304 non-HCQ group) extracted from one controlled clinical trial [22] and five observational studies $[4,13,14,31,32]$ were pooled. The overall random effect analysis indicated HCQ therapy did not appear to halt disease progression $(\mathrm{OR}=0.9 ; 95 \% \mathrm{CI}$ : $0.36-2.29)$. The test result for the overall effect, $Z=0.21$ $(p=0.83)$. This finding was also replicated by subgroup analysis of observational studies $(\mathrm{OR}=1.15 ; 95 \% \mathrm{CI}$ : 0.45-2.95). However, in subgroup analysis, the finding from a single controlled clinical trial $(\mathrm{OR}=0.17 ; 95 \% \mathrm{CI}$ : 0.04-0.86) was in favor of the HCQ group (Figure 5). Though the study had no risk of bias, the small sample size (31 in each arm) used made the interpretation of the finding extremely tricky. Several studies also indicated HCQ may not improve the rate of disease progression in COVID-19 patients. In a controlled clinical trial [8], which was not included in the pooled analysis, 3 patients were progressed to severe disease in the HCQ group (3/14 versus 0/14). Moreover, in three noncomparative studies [7, 17, 21, 22], out of 1,192 COVID-19 patients treated with HCQ with or without AZ, 42 patients were transferred to ICU and intubated.

5.4. Adverse Effects. The data from four controlled clinical trials [8, 20-22] of 278 COVID-19 patients (141 HCQ and 137 from the non-HCQ group) were included to assess overall adverse effects (except QTc prolongation) among HCQ-exposed patients. Three controlled clinical trials [20-22] were pooled, and one controlled clinical trial [8] was described narratively due to its risk of bias A. In the pooled analysis, the odds of adverse effects among COVID-19 patients treated with HCQ patients were increased by 2.35 $(\mathrm{OR}=2.35 ; 95 \% \mathrm{CI}: 1.15-4.8)$. The test for the overall effect 
TABLE 1: Characteristics of the studies included in the meta-analysis

\begin{tabular}{|c|c|c|c|c|c|}
\hline Author & Study design & Population & $\begin{array}{l}\text { Intervention group and } \\
\text { protocol }\end{array}$ & Control & Outcome \\
\hline $\begin{array}{l}\text { Gautret et al., } \\
2020[8]\end{array}$ & $\begin{array}{l}\text { Open-label } \\
\text { nonrandomized } \\
\text { clinical trial }\end{array}$ & Age $>12$ & $\begin{array}{c}\text { HCQ: } 20 \\
\text { Protocol: } 200 \mathrm{mg} \text { tid/10 } \\
\text { days }\end{array}$ & $\begin{array}{l}\text { Non-HCQ: } \\
16\end{array}$ & $\begin{array}{c}\text { Viral cure on day } 6 \text { (14/20 vs. } 2 / 16) \text { : } \\
\text { HCQ group } 8 / 14 \text { and HCQ + AZ 6/6. } \\
\text { Mortality: } 1 / 14 \text { vs. none; ICU } \\
\text { admission: } 3 / 14 \text { vs. none. Adverse } \\
\text { effects: } 1 / 14 \text { (nausea) vs. none }\end{array}$ \\
\hline $\begin{array}{l}\text { Barbosa Espe } \\
\text { et al., } 2020[32]\end{array}$ & & Age $>18$ & $\begin{array}{c}\text { HCQ: } 412 \\
\text { Protocol: } 800 \mathrm{mg} / \text { on day } 1 \text {, } \\
400 \mathrm{mg} / 6 \text { days and AZ } \\
500 \mathrm{mg} / \text { day } / 5 \text { days } \\
\text { HCQ group: } 75\end{array}$ & $\begin{array}{l}\text { Non-HCQ: } \\
224\end{array}$ & $\begin{array}{l}\text { Need for hospitalization: HCQ + AZ, 8/ } \\
\qquad 412 \text { vs. } 12 / 224\end{array}$ \\
\hline $\begin{array}{l}\text { Tang et al., } \\
2020 \text { [20] }\end{array}$ & & Age $\geq 18$ & $\begin{array}{l}\text { Protocol: } 1.2 \mathrm{gd} \mathrm{LD} / \mathrm{d} / 3 \\
\text { days, and then, } 800 \mathrm{mg} / \mathrm{d} \\
\text { for } 2-3 \text { weeks }\end{array}$ & $\begin{array}{l}\text { Non-HCQ: } \\
75\end{array}$ & $\begin{array}{l}\text { Viral cure on day } 28: 53 / 75 \text { vs. } 56 / 75 \text {; } \\
\text { adverse effect: } 21 / 75 \text { vs. } 7 / 80\end{array}$ \\
\hline $\begin{array}{l}\text { Jun et al., } 2020 \\
\text { [21] }\end{array}$ & & Age $\geq 18$ & $\begin{array}{c}\text { HCQ: } 15 \\
\text { Protocol: HCQ } 400 \mathrm{mg} \text { qod } \\
\text { 5/days }\end{array}$ & $\begin{array}{c}\text { Non-HCQ: } \\
15\end{array}$ & $\begin{array}{c}\text { Viral cure after day } 7: 13 \text { vs. } 14 \text {; adverse } \\
\text { effects: } 4 / 15 \text { vs. } 3 / 15\end{array}$ \\
\hline $\begin{array}{l}\text { Chen et al., } \\
2020 \text { [22] }\end{array}$ & & Age $\geq 18$ & $\begin{array}{c}\text { Non-HCQ: } 15 \\
\text { Protocol: } 400 \mathrm{mg} / \mathrm{d} / 5 \text { days }\end{array}$ & $\begin{array}{l}\text { Non-HCQ } \\
\text { group: } 31\end{array}$ & $\begin{array}{l}\text { Clinical deterioration: } 2 \text { vs. } 9 ; \\
\text { progression to severe illness: } 0 \text { vs. } 4\end{array}$ \\
\hline $\begin{array}{l}\text { Geleris et al., } \\
2020 \text { [4] }\end{array}$ & $\begin{array}{c}\text { Prospective } \\
\text { observational study }\end{array}$ & Adults & $\begin{array}{c}\text { HCQ: } 811 \text { protocol: } 600 \mathrm{mg} \\
\text { bid day } 1 \text { and then } 400 \mathrm{mg} \\
\text { daily for a median of } 5 \\
\text { days) }\end{array}$ & $\begin{array}{l}\text { Non-HCQ: } \\
562\end{array}$ & $\begin{array}{c}\text { Mortality: HCQ, 157/811; non-HCQ, } \\
\text { 75/565 } \\
\text { Intubated: 154/811; non-HCQ, 26/565 }\end{array}$ \\
\hline $\begin{array}{l}\text { Rosenberg } \\
\text { et al., } 2020[14]\end{array}$ & Retrospective cohort & All patients & $\begin{array}{c}\text { HCQ + AZ: } 735 \\
\text { HCQ: } 271 \\
\text { Protocol: not clear! }\end{array}$ & $\begin{array}{l}\text { Non- } \\
\mathrm{HCQ}+\mathrm{AZ} \\
221\end{array}$ & $\begin{array}{l}\text { Mortality: HCQ + AZ: } 189 / 735 \text {; HCQ: } \\
\text { 54/271; none: } 28 / 221 \\
\text { ICU admission: HCQ: } 52 \text {, non-HCQ: } \\
\text { 27; HCQ + AZ: } 226 / 735 \\
\text { QTc prolongation: HCQ + AZ: 81; } \\
\text { HCQ: 39; none: } 15 \\
\text { Arrhythmia: HCQ + AZ: } 150 \text {; HCQ:44; } \\
\text { and none: } 23 \\
\text { Cardiac arrest: HCQ + AZ:114; HCQ: } \\
\text { 37; none:13 }\end{array}$ \\
\hline $\begin{array}{l}\text { Mercuro et al., } \\
2020 \text { [33] }\end{array}$ & Retrospective cohort & & HCQ + AZ: 53 & $\begin{array}{l}\text { Non-HCQ } \\
\text { alone: } 37\end{array}$ & $\begin{array}{c}\text { QTc prolongation: HCQ + AZ: 11/53 } \\
\text { and HCQ: 7/37 }\end{array}$ \\
\hline $\begin{array}{l}\text { Saleh et al., } \\
2020[26]\end{array}$ & Prospective study & & HCQ + AZ: 119 & $\begin{array}{c}\text { Non-HCQ: } \\
191\end{array}$ & $\begin{array}{c}\text { QTc prolongation: HCQ + AZ: 11/119 } \\
\text { and HCQ: 7/191 }\end{array}$ \\
\hline $\begin{array}{l}\text { Mahévas et al., } \\
2020 \text { [13] }\end{array}$ & $\begin{array}{c}\text { Comparative } \\
\text { observational study }\end{array}$ & $\begin{array}{l}\text { Age } \geq 18 \\
\text { years }\end{array}$ & $\begin{array}{c}\text { HCQ: } 84 \\
\text { Protocol: } 600 \text { mg within } 48 \\
\text { hours of admission }\end{array}$ & $\begin{array}{l}\text { Non-HCQ: } \\
89\end{array}$ & $\begin{array}{l}\text { Mortality: } 9 / 84 \text { vs. } 8 / 89 . \text { ICU } \\
\text { admission: } 8 / 84 \text { vs. } 14 / 89\end{array}$ \\
\hline $\begin{array}{l}\text { Yu et al., } 2020 \\
\text { [30] }\end{array}$ & $\begin{array}{l}\text { Retrospective } \\
\text { observational }\end{array}$ & $\begin{array}{c}\text { Not } \\
\text { specified }\end{array}$ & $\begin{array}{c}\text { HCQ: } 48 \\
\text { Protocol:200 mg bid/7-10 } \\
\text { days }\end{array}$ & $\begin{array}{c}\text { Non-HCQ: } \\
520\end{array}$ & Mortality: $9 / 48$ vs. $238 / 520$ \\
\hline $\begin{array}{l}\text { Magagnoli } \\
\text { et al., } 2020[31]\end{array}$ & $\begin{array}{l}\text { Retrospective } \\
\text { observational }\end{array}$ & $\begin{array}{c}\text { Not } \\
\text { specified }\end{array}$ & $\begin{array}{c}\text { HCQ: } 97 \\
\text { HCQ + AZ: } 113\end{array}$ & $\begin{array}{c}\text { Non-HCQ: } \\
158\end{array}$ & $\begin{array}{l}\text { Mortality: HCQ: 27; HCQ + AZ: 25; no } \\
\text { HCQ: 18. ICU admission/need for } \\
\text { ventilation: HCQ: } 12 / 90 \text {; HCQ + AZ: } 7 / \\
\text { 101; no HCQ: } 25 / 177\end{array}$ \\
\hline
\end{tabular}

was statistically significant $(p=0.02)$, and the summary effect of the meta-analysis was heterogeneity: $\mathrm{Chi}^{2}=3.91$, $\mathrm{d} f=3(p=0.27) ; I^{2}=23 \%$ (Figure 6).

Besides, Gautret et al. [8] found more adverse effects among patients randomized to HCQ (1/14 versus $0 / 16)$.

5.5. QTc Prolongation. QTc prolongation was reported in two ways in most of the studies. The cut-off points, an increase in greater than $60 \mathrm{~ms}$ from baseline, and absolute QTc increase to greater than $500 \mathrm{~ms}$ were used as a threshold to discontinue medications responsible or suspected to cause QTc prolongation. These cut-off points were described by recent guidelines and FDA $[18,35]$. An absolute QTc prolongation greater than $500 \mathrm{~ms}$ was reported in two observation studies [26, 31], which compared HCQ alone with HCQ plus AZ. A study by Rosenberg et al. [14] reported that after ECG screening, 80 patients $(12.6 \%)$ had a QTc prolongation in the combination group, whereas 39 patients (16.7\%) developed QTc prolongation in the HCQ group.

In the current review, the data of 291 patients from two observational studies $[26,31]$ were pooled to estimate the 
TABLE 2: Characteristics of the studies included in the meta-proportion.

\begin{tabular}{|c|c|c|c|c|}
\hline Authors & Study design & Population & Intervention & Outcomes \\
\hline $\begin{array}{l}\text { Molina } \\
\text { et al., } \\
2020[12]\end{array}$ & $\begin{array}{c}\text { Prospective, } \\
\text { nonrandomized, } \\
\text { noncomparative } \\
\text { open-labeled } \\
\text { study }\end{array}$ & $\begin{array}{l}\text { (i) } 11 \text { patients } \\
\text { (ii) Mean age } 58.7 \\
\text { years }\end{array}$ & $\begin{array}{l}\text { HCQ } 600 \mathrm{mg} / \text { day for } 10 \\
\text { days and + AZ } 500 \mathrm{mg} \\
\text { on day } 1 \text { and then } \\
250 \mathrm{mg} \text { on days } 2-5\end{array}$ & $\begin{array}{l}\text { After } 5 \text { days, } \\
\text { (i) One died } \\
\text { (ii) Two } \\
\text { transferred to } \\
\text { the ICU }\end{array}$ \\
\hline
\end{tabular}

Adverse effects of
HCQ Remark

(i) Very small sample size 1 patient (ii) Risk factors discontinued drugs after 4 days due to QT interval prolongation from $405 \mathrm{~ms}$ before treatment to 460 and $470 \mathrm{~ms}$ under the combination

for QTc

prolongation

were not

addressed death was not stated (iii) The cause of

(iv) The severity of the disease was not stated

(i) 25 reported mild adverse events. 3 patients discontinued treatment (due to abdominal pain, urticaria, (i) 1061 patients:
Other drugs suspected to affect QT were systematically stopped mean age 43.6 years (14-95 HCQ $200 \mathrm{mg}$ TID for 10 days

Million

et al., Retrospective years) report

(ii) The majority (95.0\%) of $\mathrm{HCQ}+\mathrm{AZ}$ (500 mg on 2020 [11] day 1 followed by ICU: 10 patients had a low 4 days) for at least 3 days failure) NEWS score

erythematous, and bullous rash)

(ii) 9 patients had a

QTc prolongation of more than $60 \mathrm{~ms}$ from baseline but no patient exceeded $500 \mathrm{~ms}$. No rhythmic, cardiac events or sudden deaths. None showing torsade de pointe

(i) TDPs due to $\uparrow Q T c=0$ patients HCQ: $191(95.0 \%)$

(i) 210 patients patients

$\begin{array}{lcl}\text { Saleh } & \text { Prospective } & \text { (ii) Mean age: } \\ \text { et al., } & \text { observational } & 58.5 \pm 9.1 \mathrm{yrs} \\ \text { 2020 [26] } & \text { study } & \text { (iii) Baseline QTc: } \\ & & 439.5 \pm 24.8\end{array}$

(i) HCQ $400 \mathrm{mg}$ PO BID for one day followed by $200 \mathrm{mg}$ PO BID for 4 days \pm AZ $500 \mathrm{mg}$ PO or IV daily for 5 days to 119 (59.2\%) patients

(ii) 18 patients: $\uparrow \mathrm{QTc}$ ( $\geq 500 \mathrm{~ms}$ )

(iii) Arrhythmogenic death $=0$ patients (iv) $7(3.5 \%)$ patients discontinued $\mathrm{HCQ} \pm \mathrm{AZ}$ due to $\uparrow Q T c$

(i) $1 \mathrm{TdP}$ developed after extreme QTC prolongation
(ii) $40(15.9 \%)$

251 patients median age: $64 \pm 13$; baseline QTc (ms): $439 \pm 29$ (i) HCQ $400 \mathrm{mg}$ BID for one day followed by $200 \mathrm{mg}$ BID for 4 days $\mathrm{HCQ}+\mathrm{AZ}$ was given orally at a dose of $500 \mathrm{mg}$ daily for 5 days
$44(17.5 \%)$ died of respiratory or multiorgan failure (ii) $40(15.9 \%)$

patients' extreme QTC prolongation (>500 ms)

(iii) Change from baseline by $>60 \mathrm{~ms}$, occurred in 51 (20.3\%) patients. (iv) 7 extreme QTcpts discontinued a drug (i) 8 patients (4.0\%) had a baseline QTc> $500 \mathrm{~ms}$

(ii) Receiving other QTprolonging medications (81/ 210patients)

2020 [27] study

(n)


TABle 2: Continued.

\begin{tabular}{|c|c|c|c|c|c|c|}
\hline Authors & Study design & Population & Intervention & Outcomes & $\begin{array}{c}\text { Adverse effects of } \\
\text { HCQ }\end{array}$ & Remark \\
\hline $\begin{array}{l}\text { Chorin } \\
\text { et al. } 2020 \\
{[16]}\end{array}$ & $\begin{array}{c}\text { Retrospective } \\
\text { study }\end{array}$ & $\begin{array}{l}\text { (i) } 84 \text { patients. } \\
\text { Mean: } 63 \pm 15 \\
\text { years. } \\
\text { (ii) Baseline } \\
\text { average of QTc: } \\
435 \pm 24 \mathrm{~ms}\end{array}$ & $\begin{array}{l}\text { (i) HCQ } 400 \mathrm{mg} \text { BID on } \\
\text { the first day, followed by } \\
200 \mathrm{mg} \text { BID for } 5 \text { days } \\
\text { HCQ+ AZ } 500 \mathrm{mg} \text { per } \\
\text { day for } 5 \text { days }\end{array}$ & $\begin{array}{l}\text { (i) } 4 \text { patients } \\
\text { died from } \\
\text { multiorgan } \\
\text { failure, without } \\
\text { evidence of } \\
\text { arrhythmia and } \\
\text { severe QTc } \\
\text { prolongation } \\
\text { (ii) } 64 \text { remained } \\
\text { in hospital at } \\
\text { the end } \\
\text { (iii) } 16 \\
\text { discharged }\end{array}$ & $\begin{array}{l}\text { (i) } 9(11 \%) \text { patients } \\
\text { QTc prolonged to } \\
>500 \mathrm{~ms} \\
\text { (ii) } 10(12 \%) \text { patients } \\
\text { had increased }>60 \mathrm{~ms} \\
\text { (iii) No tdp events }\end{array}$ & $\begin{array}{l}\text { Receiving other } \\
\text { suspected QTc } \\
\text { prolonging drugs, } \\
32(39 \%) \text { patients }\end{array}$ \\
\hline
\end{tabular}

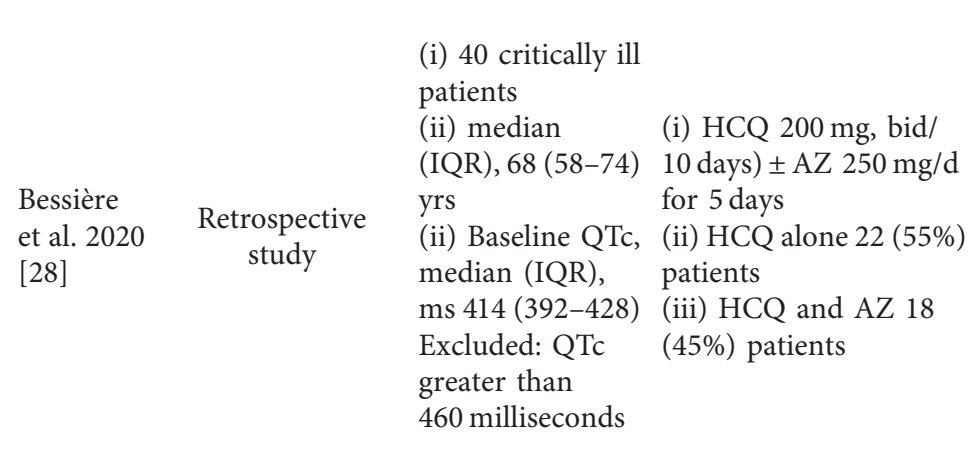

$\begin{array}{lcl} & & \text { (i) } 80 \text { patients } \\ & & \text { (ii) Median: 52.5 } \\ & \text { (18-88) years } \\ & & \text { (iii) } 92 \% \text { low } \\ \text { Gautret } & \text { Prospective } & \text { NEWS score } \\ \text { et al. } 2020 & \text { observational } & \text { (iv) QTc } \\ {[8]} & \text { study } & \text { prolonging } \\ & & \text { medical } \\ & & \text { conditions and } \\ & & \text { drugs excluded at } \\ & & \text { baseline }\end{array}$

(i) 80 patients

(ii) Median: 52.5

(18-88) years

NEWS score

(iv) QTc

prolonging

conditions and

baseline (i) After 2-5 days

QTc $\geq 500 \mathrm{~ms}$ or

$\Delta \mathrm{QTc}>60 \mathrm{~ms}(n=14$

After treatment patients)

initiation, $30 \quad$ (ii) No ventricular

(75\%) patients arrhythmia, including

torsade de pointes

The antiviral

invasive

mechanical

ventilation treatment ceased

before completion for

7 patients $(17.5 \%)$

following ECG

abnormalities (i) Lack of generalizability beyond the ICU (ii) Use of other QTc prolonging drugs (propofol, amiodarone, ciprofloxacin, and ondansetron), 20 (50\%) patients
Transfer to ICU: 3 (3.8\%), where 2 were improved and 1 HCQ $200 \mathrm{mg}$ of PO, TID for 10 days. HCQ + azithromycin $500 \mathrm{mg}$ on D1 followed by $250 \mathrm{mg}$ per day for the next 4 days

IDW. Death: 1 returned to

(1.2\%).

Discharged/

improved: 65

(81.2\%);

currently hospitalized: 14

( 1 in ICU and

13 in IDW) (i) Possible adverse events: 7 (8.7\%)

(ii) Nausea or vomiting: 2 (2.5\%)

(iii) Diarrhea 4 (5.0\%) Blurred vision: 1 (1.2\%) (i) Patients

followed-up for at least six days were included in analysis (ii) Max. 10 days Lost to follow-up patients were not known

\section{(i) 22 patients \\ (ii) Median age: \\ 64 \\ (iii) Baseline}

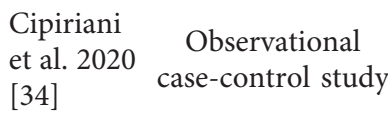

QTc-interval: 426 HCQ $200 \mathrm{mg}$ BID and

(403-447) ms

Controls: 34

health

individuals,

matched for age

and sex (i) QTc prolongation (QTc $\geq 480 \mathrm{~ms}$ ) after HCQ treatment: 4 (18\%) patients, of which 1 patient developed $>500 \mathrm{~ms}$

(ii) No cases of syncope, fatal arrhythmias, and sudden cardiac death (i) Conditions predisposing to QTc prolongation including medications were excluded

(ii) There was a significant QTc difference after HCQ initiation (426 vs. $450 \mathrm{~ms}$, $p=0.02$ )

(iii) Mean of QTc of patients was 453 (439-477) ms while of controls was 407 (397-418); $P$ value $<0.001$.

HCQ, hydroxychloroquine; AZ, azithromycin; CQ, chloroquine; IDW, infectious disease ward; NEWS, National Early Warning Score; $\uparrow$, increase; PO, oral; BID, twice daily; ms, milliseconds. Discontinuation of a subject from a clinical trial should be considered if there is an increase in QT/QTc to $>500 \mathrm{~ms}$ or if $>60 \mathrm{~ms}$ over baseline are commonly used as thresholds for potential discontinuation. Guidance for Industry E14 Clinical Evaluation of QT/QTc Interval Prolongation and Proarrhythmic Potential for Non-Antiarrhythmic Drugs. 


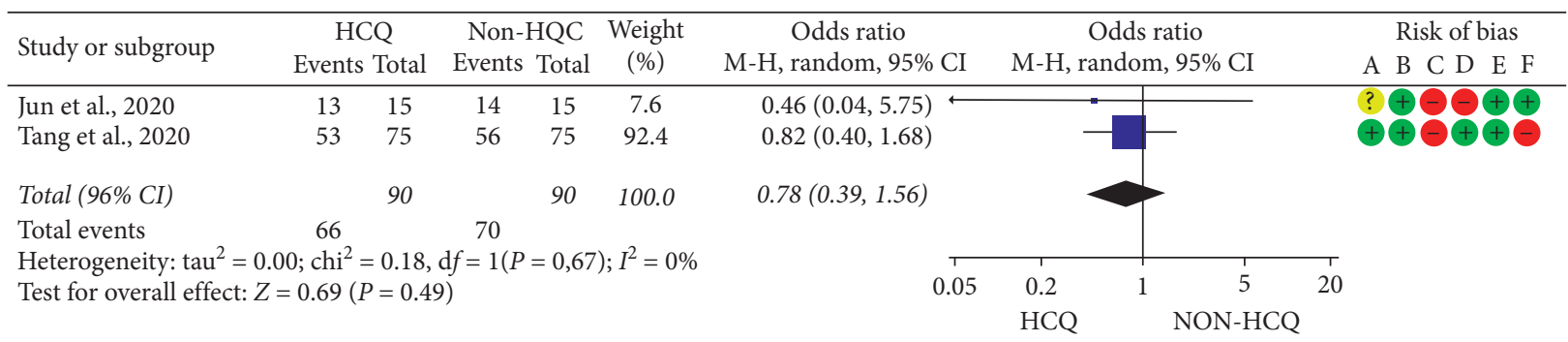

$\underline{\text { Risk of bias legend }}$

(A) Random sequence generation (selection bias)

(B) Allocation concealment (selection bias)

(C) Blinding of participants and personnel (performance bias)

(D) Blinding of outcome assessement (detection bias)

(E) Incomplete outcome data (attrition bias)

(F) Selective reporting (reporting bias)

FIgURe 2: Virologic efficacy of hydroxychloroquine (HCQ) as compared to non-HCQ for patients with COVID-19.

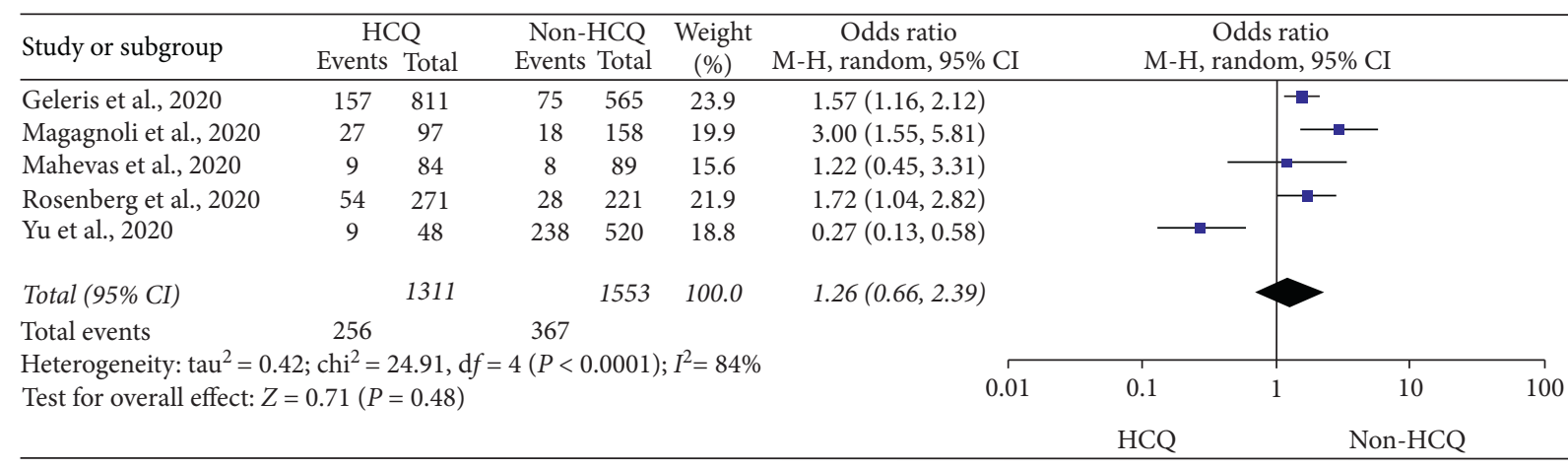

FIgURE 3: Risk of mortality among COVID-19 patients exposed to HCQ as compared to non-HCQ (standard care).

Studies

Molina et al., 2020

Million et al., 2020

Chorin et al., 2020

Chorin et al., 2020

Gautret et al., 2020

Overall $\left(I^{2}=92.28 \%, P<0.001\right)$

\begin{tabular}{ll}
\multicolumn{2}{c}{ Estimate $(95 \% \mathrm{CI})$} \\
$0.091 \quad(0.000,0.261)$ \\
0.008 & $(0.002,0.013)$ \\
0.175 & $(0.128,0.222)$ \\
0.048 & $(0.002,0.093)$ \\
0.012 & $(0.000,0.037)$ \\
0.058 & $(0.009,0.108)$
\end{tabular}

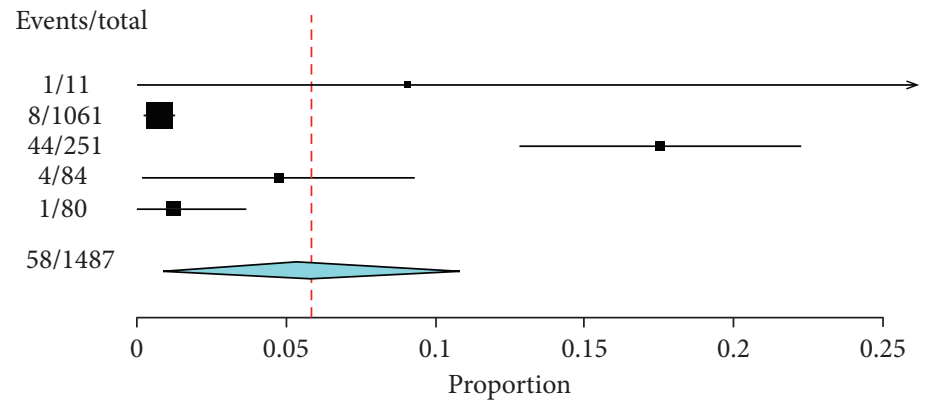

FIgURE 4: The pooled prevalence of mortality among COVD-19 patients treated with HCQ with or without AZ.

risk of QTc prolongation in patients exposed to HCQ versus HCQ plus AZ.

The result indicated the risk of QTc prolongation above $500 \mathrm{~ms}$ due to HCQ was statistically not different from those receiving HCQ plus AZ (OR = 1.11; 95\% CI: 0.54-2.28) with the test for the overall effect of $Z=0.27(p=0.79)$; heterogeneity: $\mathrm{Chi}^{2}=0.00, \mathrm{df}=1(p=0.97) ; I^{2}=0 \%$ (Figure 7$)$.

However, the findings from noncomparative studies appeared much concerning. Seven single-arm studies reported data on QT prolongation after $\mathrm{HCQ} \pm \mathrm{AZ}$ exposure. Six studies (1,657 patients) were reported a baseline increase in QTc by more than $60 \mathrm{~ms}[11,12,16,26-28]$ after $\mathrm{HCQ} \pm \mathrm{AZ}$ exposure. The result of the analysis showed about
$13.0 \%$ (95\% CI: $3.8 \%-22 \%$ ) of patients had an increase of QTc by more than $60 \mathrm{~ms}$ from the baseline. Considerable heterogeneity was present between studies $(Q=104.16$, $I^{2}=95.2 \%, p<0.001$ ) (Figure 8). Several studies also raised concerns regarding QTc prolongation following HCQ exposure with or without other medications. In four studies [16, 26-28], concomitant use of other QT-prolonging medications was reported (Table 2). Million et al. [11] and Mehta et al. [36] have excluded other drugs suspected to cause QTc prolongation at baseline. Molina et al. [12] did not address the details.

A study by Mehta et al. [36] reported 4 among 22 patients had developed QTc greater than $480 \mathrm{~ms}$ after initiation 


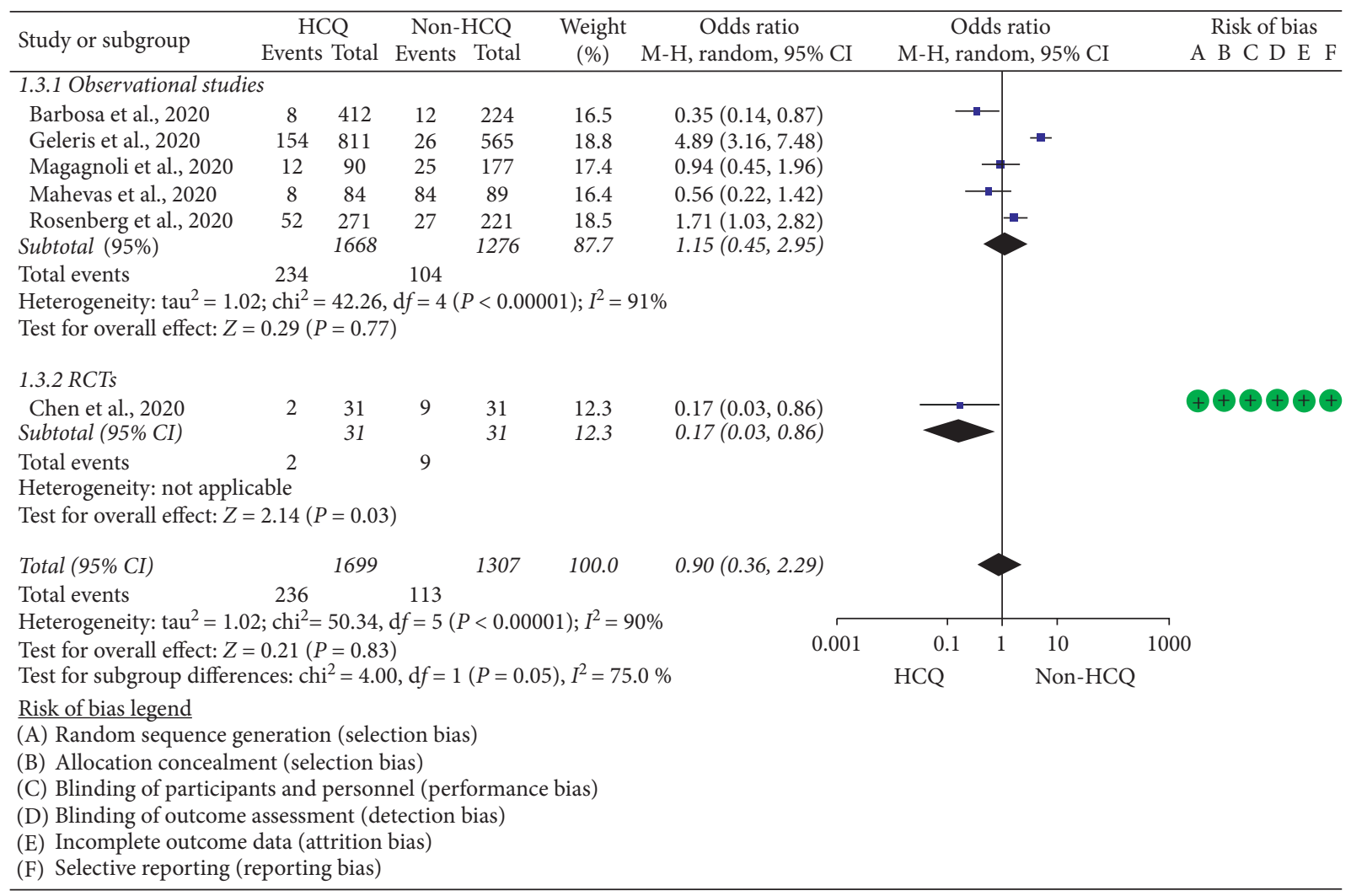

FIGURE 5: Risk of disease progression among COVID-19 patients exposed to HCQ versus non-HCQ.

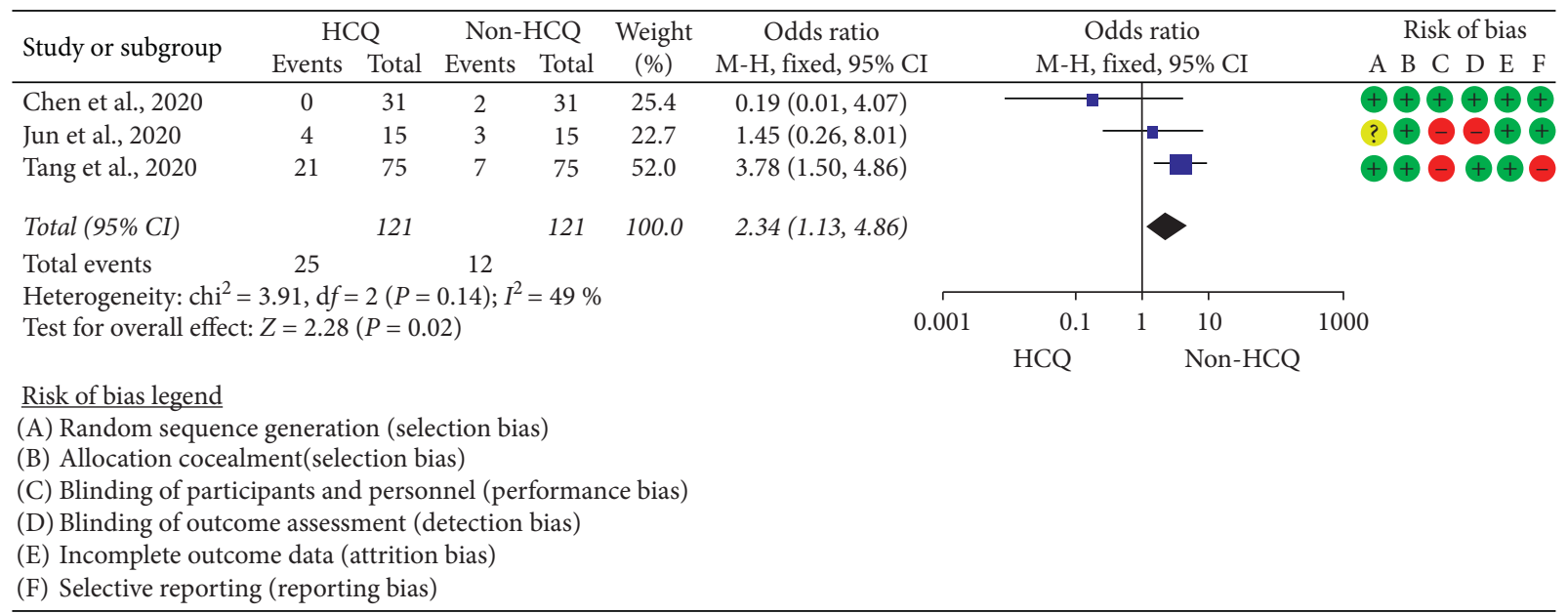

FIGURE 6: Safety of HCQ as compared to non-HCQ (standard care) among COVID-19 patients.

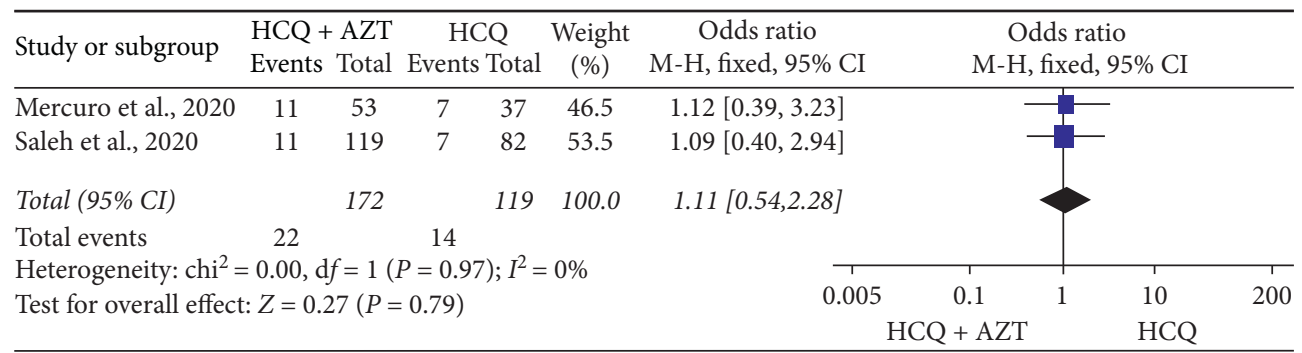

FIgURE 7: Risk of QT prolongation among COVID-19 treated with HCQ + AZ versus HCQ. 


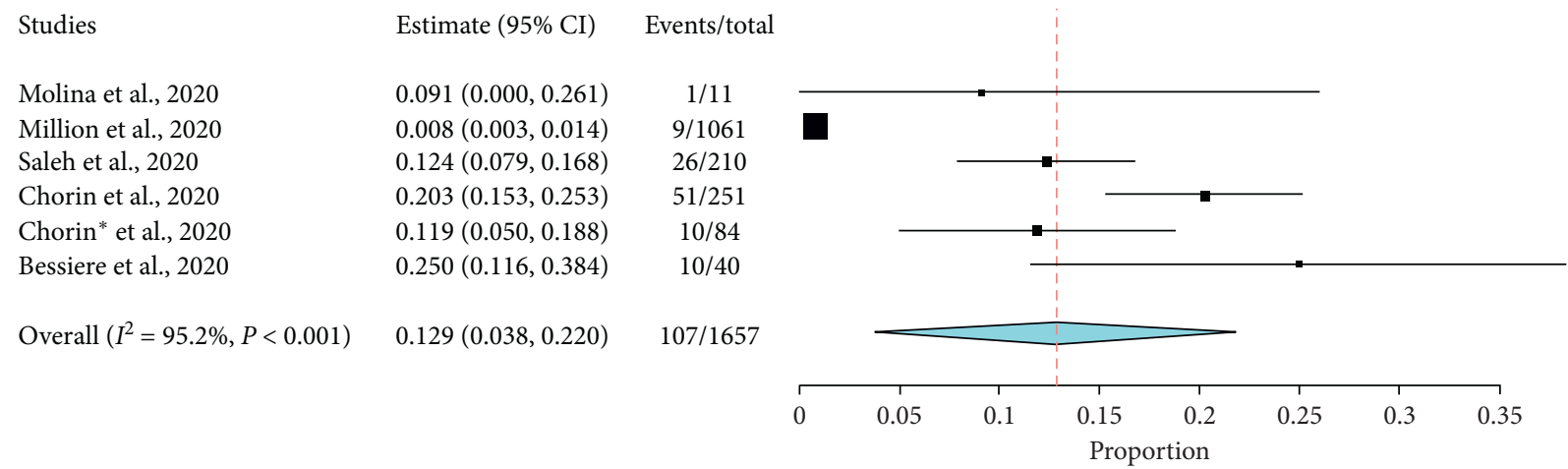

FIgURE 8: The proportion of COVD-19 patients with QTc increase by more than $60 \mathrm{~ms}$ from baseline after treatment with HCQ with or without AZ.

of therapy. Similarly, from five single-arm studies $[16,26-28,36]$, which comprised of 607 COVID-19 patients treated with $\mathrm{HCQ} \pm \mathrm{AZ}$, about $11.2 \%$ (95\% CI: 7.0\%-15.5\%) of patients had developed an absolute increase of QTc greater than $500 \mathrm{~ms}$ (Figure 9). However, a study by Million et al. [11] reported there were no patients who had an increased QTc greater than $500 \mathrm{~ms}$.

A meta-regression was conducted to assess the effect of baseline use of other drugs suspected to cause QTc prolongation to above $500 \mathrm{~ms}$. Indeed, the result did not show any significant effect (coefficients $(\mathrm{Q})=-0.0 ; 95 \% \mathrm{CI}$ : $-0.002-0.000 ; p=0.79$ ).

In turn, the pooled prevalence of drug discontinuation due to the increased QTc prolongation to greater than $500 \mathrm{~ms}$ in three studies [26-28] and to greater than $60 \mathrm{~ms}$ from baseline in one study [12] among COVID-19 patients treated with HCQ with or without AZ was 4.1\% (95\% CI: 1.1-7.1). The heterogeneity among the included studies was also acceptable enough $\left(I^{2}=52.19, p=0.099\right)$ (Figure 10). In one study [11], three patients had discontinued the treatment due to adverse events other than QTc prolongation.

5.6. Admission to the Intensive Care Unit (ICU) or Death. The data of 2,413 (729 HCQ and 1684 HCQ + AZ) COVID19 patients were included to estimate the independent effect of AZ on mortality and ICU admission $[14,31]$. Accordingly, HCQ plus AZ did not improve the risk of mortality $(\mathrm{OR}=0.93 ; 95 \% \mathrm{CI}: 0.51-1.72)$ and ICU admission $(\mathrm{OR}=0.97 ; 95 \% \mathrm{CI}: 0.26-3.61)$. The overall pooled result showed a statistically insignificant result for the composite outcome $(\mathrm{OR}=0.88 ; 95 \% \mathrm{CI}: 0.55-1.43)$. The test for the overall effect was $Z=0.50(p=0.61)$, and the heterogeneity was $\mathrm{Tau}^{2}=0.16 ; \mathrm{Chi}^{2}=11.3, \mathrm{~d} f=3 \quad(p=0.01) ; I^{2}=73 \%$ (Figure 11).

\section{Discussion}

The global community is in the state of urgency to mitigate the health and economic crisis instigated by COVID-19. Chloroquine (CQ) and its derivative hydroxychloroquine (HCQ) have traditionally been used for the treatment of malaria and certain autoimmune diseases. The drugs have possible activity against SARS-CoV-1 and SARS-CoV-2 in vitro and in clinical practice [36]. However, clinical studies were reporting contradictory results on the efficacy and safety of HCQ when used for treating COVID-19 patients. Thus, systematic review and meta-analysis of the existing studies was performed to explore the efficacy, safety, and tolerability of HCQ with or without AZ in COVID-19 patients.

The finding of this meta-analysis suggested the use of HCQ did not result in a rapid viral clearance. It also failed to improve survival and rate of disease progression. The pooled prevalence of mortality was higher in patients exposed to HCQ with or without AZ. Moreover, HCQ exposure carried a significant risk of adverse effects and a sizable proportion of patients ended up with drug discontinuation.

On the other hand, a combination of HCQ and AZ did not result in increased risk of QTc prolongation, improved survival, or preventing admission to ICU. However, the pooled proportion of observational studies indicated an alarming rate of QTc prolongation among patients receiving HCQ with or without AZ.

The finding of this study showed an absence of rapid viral clearance $(\mathrm{OR}=0.78 ; 95 \% \mathrm{CI}: 0.39-1.56)$ in patients treated with HCQ compared to the standard care. However, the review by Yang et al. [35] found better virologic efficacy with statistically insignificant results. The finding of this review was based on the data from a single study [8], which had a high risk of bias. More importantly, the former review compared HCQ + AZ with the standard care (control group). This arm of the study had a virologic cure rate of $100 \%$ reported from 6 patients. Nevertheless, the current review considered the arm which compared HCQ alone versus the control group, which reported a virologic cure rate of $57 \%$ in the HCQ arm versus $12.5 \%$ in the control arm. The study by Shamshirian et al. [37] had also questioned the virologic potency of $\mathrm{HCQ}+\mathrm{AZ} \quad(\mathrm{RR}=2.15,95 \% \mathrm{CI}$ : $0.31-14.77)$. Moreover, the optimal duration for virologic clearance is not well known. Gautret et al. [8], Jun et al. [21], and Tang et al. [20] reported a virologic cure after 6, 7, and 28 days of treatment, respectively. Therefore, drawing a conclusion from such findings irrespective of the incurred heterogeneity may be erroneous. Of note, all the included studies were open-labeled, non-randomized, and the authors did not describe the type of treatment given for the control group. 
Studies

Saleh et al., 2020

Chorin et al., 2020

Chorin* et al., 2020

Bessiere et al., 2020

Cipriani et al., 2020

Overall $\left(I^{2}=57.53 \%, P<0.051\right)$
Estimate (95\% CI) Events/total

$\begin{array}{cc}0.086(0.048,0.124) & 18 / 210 \\ 0.159(0.114,0.205) & 40 / 251 \\ 0.207(0.041,0.173) & 9 / 84 \\ 0.175(0.057,0.293) & 7 / 40 \\ 0.045(0.000,0.132) & 1 / 22\end{array}$

$0.112(0.070,0.155)$

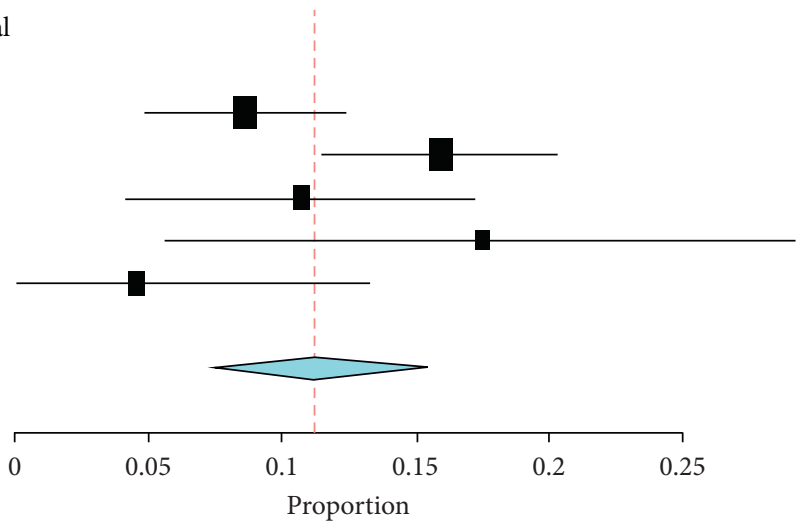

FIGURE 9: The proportion of COVD-19 patients with absolute QTc increase by more than $500 \mathrm{~ms}$ after treatment with HCQ with or without AZ.

\section{Studies}

Molina et al., 2020

Saleh et al., 2020

Chorin et al., 2020

Bessiere et al., 2020

Overall $\left(I^{2}=52.19 \%, P=0.051\right)$

Estimate $(95 \% \mathrm{CI})$
$0.091(0.000,0.261)$
$0.033(0.009,0.058)$
$0.028(0.008,0.048)$
$0.175(0.057,0.293)$
$0.041(0.011,0.071)$

Events/total
$1 / 211$
$7 / 210$
$7 / 251$
$7 / 40$
$22 / 512$

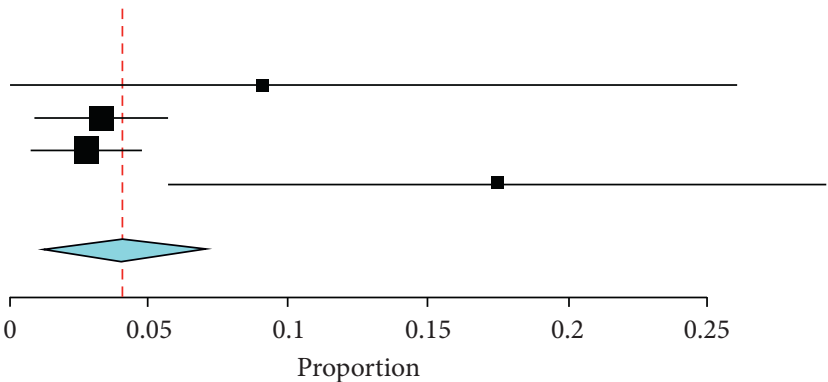

FIgURE 10: Proportion of patients' drug discontinuation due to an increase of QTC prolongation ( $>500 \mathrm{~ms})$ in COVID-19 patients treated with HCQ with or without AZ.

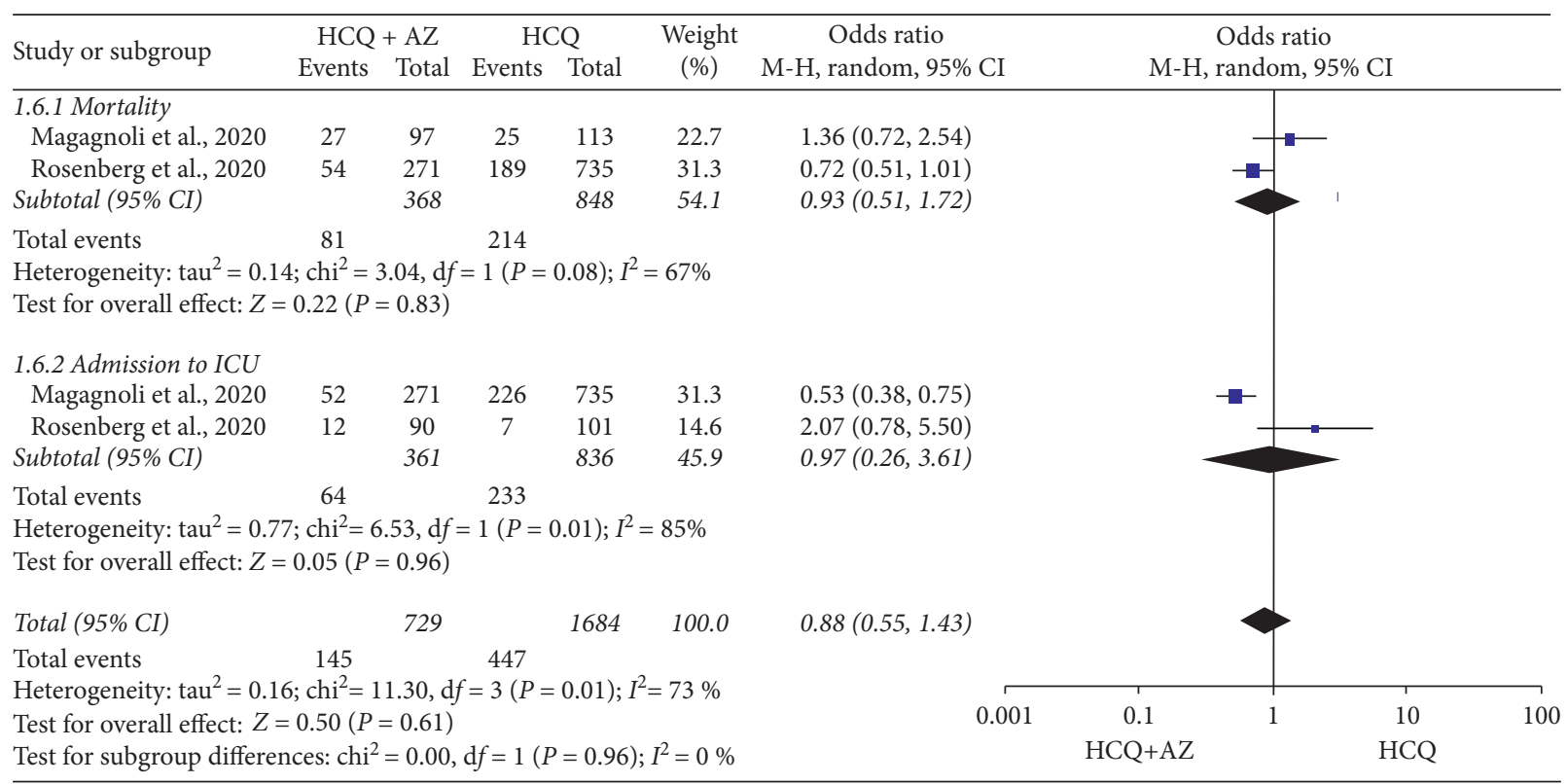

FIGURE 11: Risk of death or ICU admission in patients exposed to HCQ versus HCQ + AZ.

Similarly, HCQ could not demonstrate improved survival $(\mathrm{OR}=1.26 ; 95 \% \mathrm{CI}: 0.66-2.39)$ among COVID-19 patients. Gautret et al. [8] also reported an episode of death in the HCQ group, but not in the control group. Previous reviews [37-39] reported either increased mortality among HCQ groups or no statistically significant difference. As compared to our review, earlier reviews included a limited number of studies, while Sarma et al. [15] reported a 
combined result of mortality and clinical worsening. Our finding was not different from former studies $[15,39]$. This review also found a $5.8 \%$ pooled prevalence of mortality from five observational studies [11, 12, 16, 27, 29] among COVID-19 patients treated with HCQ with or without AZ. In three $[11,16,27]$ of these studies, respiratory and multiorgan failure was the cause of death. There was no reported death due to adverse effects. In all of the included studies, patients had active comorbidity. There was significant heterogeneity among the studies $\left(I^{2}=92.28 \%, p \leq 0.001\right)$, which could be attributed to the differences in disease severity and age of the participants. Yet, meta-regression analysis did not produce any evidence.

Furthermore, HCQ therapy was not significantly associated with slowing the composite end point of disease progression $(\mathrm{OR}=0.9 ; 95 \% \mathrm{CI}: 0.36-2.29)$, unlike the finding by Chen et al. [22] ( $\mathrm{OR}=0.17$; 95\% CI: 0.04-0.86). The finding by Chen et al. [22] may not be dependable as it included limited number of patients (31 in each arm). The subgroup analysis result of observational studies also indicated HCQ therapy was not significantly affecting disease progression $(\mathrm{OR}=1.15$; 95\% CI: 0.45-2.95). Analogous results were reported by the preceding studies $[15,38,40]$. However, one review indicated that HCQ may prevent progression to severe disease among COVID-19 patients [35]. Since the previous review included a limited number of studies, its finding must be interpreted cautiously. Our finding was in agreement with reviews by Sarma et al. [15] and Shamshirian et al. [37]. Nonetheless, these studies reported better outcomes of radiological progression in the HCQ arm, a finding generated from two poorly designed controlled trials $[21,22]$.

The safety of HCQ with or without AZ has been questioned by several studies [37, 41]. Diarrhoea, vomiting, blurred vision, rash, and headache were commonly reported adverse effects. In this review, the risk of adverse effects among COVID-19 patients treated with HCQ was 2.35 $(\mathrm{OR}=2.35$; 95\% CI: 1.15-4.8). Shamshirian et al. [37] also found an increase in the odds of the adverse effect among patients exposed to HCQ (OR=3.55, 95\% CI: 1.61-7.82). Similar findings were also reported by other studies [40-42].

However, one study reported a conflicting finding where HCQ may be safe and effective, though the authors hinted more data are required for a definitive conclusion [15]. A study conducted in China reported $37.8 \%$ of ADRs in a cluster of 217 COVID-19 patients. The predominant adverse effects were drug-induced gastrointestinal disorders and liver system disorders [43]. Cardiac side effects including conduction disturbances (bundle-branch block, incomplete or complete atrioventricular block, QT prolongation, and subsequent torsade de pointes) and cardiomyopathy (hypertrophy and congestive heart failure) were also described $[44,45]$.

In this review, although the risk of QTc prolongation above $500 \mathrm{~ms}$ among those exposed to HCQ (OR $=1.11$; 95\% CI: 0.54-2.28) may not appear concerning, HCQ/AZ combination could be more worrisome. A finding generated from five single-arm studies [16, 26-28, 36], which comprised the data of 607 COVID-19 patients treated with

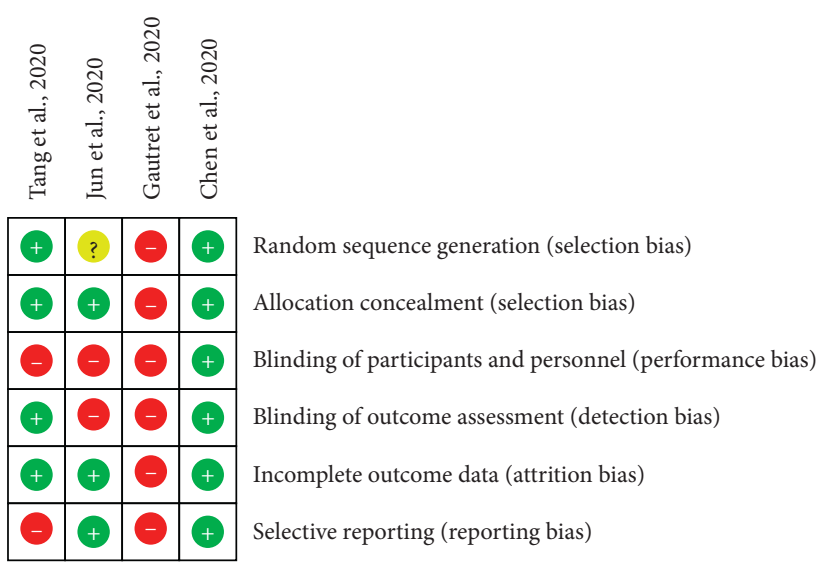

Figure 12: Risk of bias assessment for controlled clinical trials.

$\mathrm{HCQ} \pm \mathrm{AZ}$, showed $11.2 \%$ (95\% CI: $7.0 \%-15.5 \%$ ) in the pooled prevalence of QTc prolongation above $500 \mathrm{~ms}$. In addition, a finding generated from other observational studies [11, 12, 16, 26-28] indicated 13.0\% (95\% CI: 3.8\%$22 \%$ ) of patients had an increase in QTc by more than $60 \mathrm{~ms}$ from the baseline. This finding was not similar to the data synthesized from double-arm studies. This is because of the fact that the double-arm section had compared the data of HCQ alone with HCQ + AZ. As adverse cardiovascular sequels, such as myocarditis, acute myocardial infarction, and heart failure, have been reported in COVID-19 patients and these off-label therapies are not familiar to cardiovascular clinicians managing these patients [46], emergency care physicians should outweigh the risk and the benefits. In a nutshell, numerous studies $[14,16,17,26,27,46]$ have raised concerns over the cardiac safety of this combination.

Drug discontinuation due to adverse effects was also a common finding. The current study showed $4.1 \%$ of patients discontinued treatment due to an increase in QTc prolongation. Another study also indicated 4.5\% of COVID-19 patients treated with HCQ with or without AZ have discontinued treatment due to adverse effects. Similarly, one patient out of 117 discontinued HCQ in patients receiving HCQ with or without AZ after three days due to QTc prolongation [47].

Though the combination of HCQ + AZ was effective in some studies [15, 35], the other study [37] found it carries more hazard of death $(\mathrm{RR}=3.65 ; 95 \% \mathrm{CI}: 1.10-12.10)$ as compared to the control group. Our findings showed a lack of evidence for curbing mortality and intensive care unit admission of this combination $(\mathrm{OR}=0.88 ; 95 \%$ CI: $0.55-1.45)$.

It is irrefutable that our study has several limitations. The inclusion of studies with a high risk of bias and methodological flaws, combining findings from controlled and uncontrolled studies, may limit its generalizability. We also reported more than one outcome, for instance, ICU admission and need of hospitalization under a single heading, namely, disease progression. This may not show the true picture of the real-world. The existence of heterogeneity, uniform treatment of all cases (mild to severe), and inclusion of limited studies with a small sample size for outcomes such 


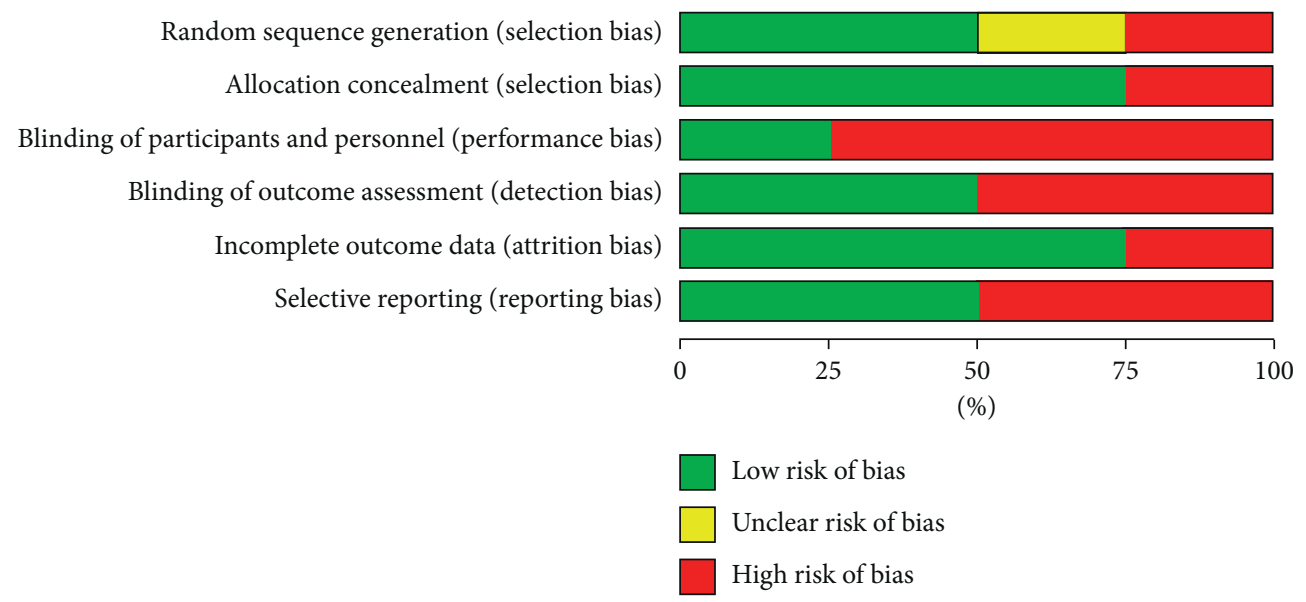

FIGURE 13: Risk of bias graph: review authors' judgments about each risk of bias item presented as percentages across all included studies.

Table 3: Modified Newcastle-Ottawa Quality Assessment Scale for the included observational studies.

\begin{tabular}{|c|c|c|c|c|c|c|c|c|c|}
\hline Selection $^{\mathrm{a}}$ & & & & & Comparability $^{\mathrm{b}}$ & & Outc & $m e^{c}$ & \\
\hline $\begin{array}{l}\text { Included } \\
\text { studies }\end{array}$ & $\begin{array}{c}\text { Representativeness } \\
\text { of the exposed } \\
\text { cohort }\end{array}$ & $\begin{array}{l}\text { Selection of } \\
\text { nonexposed }\end{array}$ & $\begin{array}{l}\text { Ascertainment } \\
\text { of exposure }\end{array}$ & $\begin{array}{l}\text { Outcome } \\
\text { of interest } \\
\text { was not } \\
\text { present at } \\
\text { start of } \\
\text { study }\end{array}$ & & $\begin{array}{l}\text { Assessment } \\
\text { of outcome }\end{array}$ & $\begin{array}{l}\text { Length } \\
\text { of } \\
\text { follow- } \\
\text { up }\end{array}$ & $\begin{array}{l}\text { Adequacy } \\
\text { of follow- } \\
\text { up }\end{array}$ & $\begin{array}{c}\text { Total } \\
\text { number } \\
\text { of stars }\end{array}$ \\
\hline $\begin{array}{l}\text { Rosenberg } \\
\text { et al., } 2020 \\
{[14]} \\
\end{array}$ & $\mathrm{A}^{*}$ & $\mathrm{~A}^{*}$ & $\mathrm{~A}^{*}$ & $\mathrm{~A}^{*}$ & $\mathrm{~A}^{*} \mathrm{~B}^{*}$ & $\mathrm{~A}^{*}$ & B & $\mathrm{A}^{*}$ & 8 \\
\hline $\begin{array}{l}\text { Mahévas } \\
\text { et al., } 2020 \\
\text { [13] } \\
\end{array}$ & $\mathrm{A}^{*}$ & $\mathrm{~A}^{*}$ & $\mathrm{~A}^{*}$ & $\mathrm{~A}^{*}$ & $A^{*} B^{*}$ & $A^{*}$ & B & $\mathrm{B}^{*}$ & 8 \\
\hline $\begin{array}{l}\text { Barbosa } \\
\text { Espe et al., } \\
2020 \text { [32] } \\
\end{array}$ & $\mathrm{A}^{*}$ & $\mathrm{~A}^{*}$ & $\mathrm{~A}^{*}$ & $\mathrm{~A}^{*}$ & $\mathrm{~B}^{*}$ & $\mathrm{~A}^{*}$ & B & $\mathrm{B}^{*}$ & 7 \\
\hline $\begin{array}{l}\text { Geleris } \\
\text { et al., } 2020 \\
{[4]} \\
\end{array}$ & $\mathrm{A}^{*}$ & $\mathrm{~A}^{*}$ & $\mathrm{~A}^{*}$ & $\mathrm{~A}^{*}$ & $\mathrm{~A}^{*} \mathrm{~B}^{*}$ & $\mathrm{~A}^{*}$ & B & $\mathrm{B}^{*}$ & 8 \\
\hline $\begin{array}{l}\text { Saleh et al., } \\
2020 \text { [26] }\end{array}$ & $\mathrm{A}^{*}$ & $\mathrm{~A}^{*}$ & $\mathrm{~A}^{*}$ & $\mathrm{~A}^{*}$ & $\mathrm{~B}^{*}$ & $\mathrm{~A}^{*}$ & B & $\mathrm{D}$ & 6 \\
\hline $\begin{array}{l}\text { Mercuro } \\
\text { et al., } 2020 \\
{[33]} \\
\end{array}$ & $\mathrm{B}^{*}$ & $\mathrm{~A}^{*}$ & $\mathrm{~A}^{*}$ & $\mathrm{~A}^{*}$ & $\mathrm{~B}^{*}$ & $\mathrm{~A}^{*}$ & B & $\mathrm{C}$ & 6 \\
\hline $\begin{array}{l}\text { Magagnoli } \\
\text { et al., } 2020 \\
{[31]} \\
\end{array}$ & $\mathrm{B}^{*}$ & $\mathrm{~A}^{*}$ & $A^{*}$ & $\mathrm{~A}^{*}$ & $\mathrm{~A}^{*} \mathrm{~B}^{*}$ & $\mathrm{~A}^{*}$ & B & $\mathrm{D}$ & 7 \\
\hline $\begin{array}{l}\text { Yu et al., } \\
2020[30]\end{array}$ & $A^{*}$ & $\mathrm{~A}^{*}$ & $\mathrm{~A}^{*}$ & $\mathrm{~A}^{*}$ & $\mathrm{~B}^{*}$ & $\mathrm{~B}^{*}$ & B & $\mathrm{D}$ & 6 \\
\hline $\begin{array}{l}\text { Mehta et al., } \\
2020 \text { [36] }\end{array}$ & $\mathrm{A}^{*}$ & $\mathrm{~A}^{*}$ & $\mathrm{~A}^{*}$ & $\mathrm{~A}^{*}$ & $\mathrm{~A}^{*} \mathrm{~B}^{*}$ & $\mathrm{~A}^{*}$ & $\mathrm{~A}^{*}$ & $\mathrm{~A}^{*}$ & 9 \\
\hline
\end{tabular}

a Selection: (1) representativeness of the exposed cohort: A, consecutive eligible participants were selected, participants were randomly selected, or all participants were invited to participate from the source population; B, not satisfying requirements in part (a) or not stated. (2) Selection of the nonexposed cohort: A, selected from the same source population;* B, selected from a different source population; C, no description. (3) Ascertainment of exposure: A, structured injury data (e.g., record completed by medical staff); $* \mathrm{~B}$, structured interview; $* \mathrm{C}$, written self-report; D, no description. (4) For a demonstration that the outcome of interest was not present at the start of the study: $*$ A, yes; B, no or not explicitly stated. ${ }^{b}$ Comparibility: for comparability of cohorts based on the design or analysis: $* \mathrm{~A}$, study controls for previous injury; $* \mathrm{~B}$, study controls for age. ${ }^{\mathrm{c} O u t c o m e: ~(1) ~ a s s e s s m e n t ~ o f ~ o u t c o m e: ~ A, ~ i n d e p e n d e n t ~ o r ~ b l i n d ~}$ assessment stated or confirmation of the outcome by reference to secure records (e.g., imaging and structured injury data); $*$ B, record linkage (e.g., identified through ICD codes on database records); $* \mathrm{C}$, self-report with no reference to original structured injury data or imaging; $\mathrm{D}$, no description. (2) Was follow-up long enough for outcomes to occur? $*$ A, yes ( $\geq 3$ months); B, no ( $<3$ months). (3) Adequacy of follow-up of cohorts: A*, complete follow-up-all participants accounted for; $* \mathrm{~B}$, subjects lost to follow-up unlikely to introduce bias $(<15 \%$ lost to follow-up, or description provided of those lost $*$ ); $\mathrm{C}$, follow-up rate $<85 \%$ and no description of those lost provided; D, no statement. ${ }^{\mathrm{d}}$ Total is out of 9 stars. Note: $\geq 7$, high-quality study; $5-7$, moderate quality study; $<5$, lowquality study. 
as virologic efficacy may result in biased findings. We could not retrieve some of the important findings such as disease severity scale for each study and the treatments used in the standard care setting.

\section{Conclusion}

This systematic review, which included a limited number of poorly designed controlled clinical trials and several real-world studies of patients with COVID-19 requiring hospitalization, found that the use of a regimen containing HCQ with or without AZ did not offer clinical benefit. HCQ with or without AZ did not improve the rate of virologic cure, disease progression, and mortality. These regimes were associated with more adverse effects. Therefore, these drug regimens should only be used in a clinical trial setting, and a large pool of data from randomized clinical trials is warranted to have concrete evidence for safety, efficacy, and tolerability [48].

\section{Appendix}

\section{A. Search Strategy}

The search strategy was created with the assistance of the librarians at the Jimma University drug information center. The Mendeley desktop was used as a reference manager.

1. PubMed. ((((2019-ncov) OR (2019 Novel corona virus)) OR (COVID-19)) OR (Coronavirus-19)) AND (((Hydroxychloroquine) OR (Plaquenil)) OR (Hydroxychloroquine sulphate))
(1) 2019-ncov
(2) 2019 Novel corona virus
(3) COVID-19
(4) Coronavirus-19
(5) Hydroxychloroquine
(6) Plaquenil
(7) Hydroxychloroquine sulphate $[\mathrm{MeSH}]$
(8) 1 OR 2 OR 3 OR 4
(9) 6 OR 7 OR 8
(10) 8 AND 9...70 articles

2. Cochrane CENTRAL. ((((2019-ncov) OR (2019 Novel corona virus)) OR (COVID-19)) OR (Coronavirus-19)) AND (((Hydroxychloroquine) OR (Plaquenil)) OR (Hydroxychloroquine sulphate))
(1) 2019-ncov
(2) 2019 Novel corona virus
(3) COVID-19
(4) Coronavirus-19
(5) Hydroxychloroquine
(6) Plaquenil
(7) Hydroxychloroquine sulphate
(8) 1 OR 2 OR 3 OR 4
(9) 6 OR 7 OR 8
(10) 8 AND 9

3. Web of Science. ((((2019-ncov) OR (2019 Novel corona virus)) OR (COVID-19)) OR (Coronavirus-19)) AND (((Hydroxychloroquine) OR (Plaquenil)) OR (Hydroxychloroquine sulphate))
(1) 2019-ncov
(2) 2019 Novel corona virus
(3) COVID-19
(4) Coronavirus-19
(5) Hydroxychloroquine
(6) Plaquenil
(7) Hydroxychloroquine sulphate
(8) 1 OR 2 OR 3 OR 4
(9) 6 OR 7 OR 8
(10) 8 AND 9

4. EMBASE. ((((2019-ncov) OR (2019 Novel corona virus)) OR (COVID-19)) OR (Coronavirus-19)) AND (((Hydroxychloroquine) OR (Plaquenil)) OR (Hydroxychloroquine sulphate))
(1) 2019-ncov
(2) 2019 Novel corona virus
(3) COVID-19
(4) Coronavirus-19
(5) Hydroxychloroquine
(6) Plaquenil
(7) Hydroxychloroquine sulphate
(8) 1 OR 2 OR 3 OR 4
(9) 6 OR 7 OR 8
(10) 8 AND 9

\section{(B) Risk of Bias Assessment Result for Included Studies}

Randomized controlled trials and the Modified NewcastleOttawa Quality Assessment Scale for the included observational studies (Figures 12 and 13 and Table 3).

\section{B.1. Randomized Controlled Trials}

B.2. Modified Newcastle-Ottawa Quality Assessment Scale for the Included Observational Studies.

\section{Data Availability}

Additional data not included in the article can be obtained from the corresponding author upon request. 


\section{Conflicts of Interest}

The authors declare that they have no conflicts of interest.

\section{Authors' Contributions}

All the authors contributed to the conceptualization and design of the research. TAM, TMF, and GZN conducted the database search, screening, quality assessment, and analysis. TAM, TMF, KKG, DDB, and GZN carried out data extraction and wrote the manuscript. KMG and DDB handled the discrepancies that arise during data extraction. All the authors read and approved the final manuscript.

\section{References}

[1] M. Park, A. R. Cook, J. T. Lim, Y. Sun, and B. L. Dickens, “A systematic review of Covid-19 epidemiology based on current evidence," Journal of Clinical Medicine, vol. 9, no. 4, p. 967, 2020.

[2] WHO Health Emergency Coronavirus 19 Dashboard, 2020.

[3] F. Zhou, T. Yu, R. Du et al., "Clinical course and risk factors for mortality of adult inpatients with Covid-19 in Wuhan, China: A retrospective cohort study," The Lancet, vol. 395, no. 10229, pp. 1054-1062, 2020.

[4] J. Geleris, Y. Sun, J. Platt et al., "Observational study of hydroxychloroquine in hospitalized patients with Covid-19," New England Journal of Medicine, vol. 382, no. 25, pp. 2411-2418, 2020.

[5] B. Cao, Y. Wang, D. Wen et al., "A trial of lopinavir-ritonavir in adults hospitalized with severe Covid-19," New England Journal of Medicine, vol. 382, no. 19, pp. 1787-1799, 2020.

[6] K. M. Kunz, "A trial of lopinavir-ritonavir in Covid-19," New England Journal of Medicine, vol. 382, no. 21, pp. 1-4, 2020.

[7] J. Dyall, R. Gross, J. Kindrachuk et al., "Middle east respiratory syndrome and severe acute respiratory syndrome: Current therapeutic options and potential targets for novel therapies," Drugs, vol. 77, no. 18, pp. 1935-1966, 2017.

[8] P. Gautret, J.-C. Lagier, P. Parola et al., "Hydroxychloroquine and azithromycin as a treatment of COVID-19: Results of an open-label non-randomized clinical trial," International Journal of Antimicrobial Agents, vol. 56, no. 1, p. 105949, 2020.

[9] M. Wang, R. Cao, L. Zhang et al., "Remdesivir and chloroquine effectively inhibit the recently emerged novel coronavirus (2019-nCoV) in vitro," Cell Research, vol. 30, no. 3, pp. 269-271, 2020.

[10] K. C. Wilson, S. H. Chotirmall, C. Bai, and J. Rello, "COVID19: Interim guidance on management pending empirical evidence. From an american thoracic society-led international task force on behalf of the international task force on COVID19," 2020, http://www.thoracic.org/professionals/clinical-.

[11] M. Million, J.-C. Lagier, P. Gautret et al., "Early treatment of COVID-19 patients with hydroxychloroquine and azithromycin: A retrospective analysis of 1061 cases in Marseille, France," Travel Medicine and Infectious Disease, vol. 35, Article ID 101738, 2020.

[12] J. M. Molina, C. Delaugerre, J. Le Goff et al., "No evidence of rapid antiviral clearance or clinical benefit with the combination of hydroxychloroquine and azithromycin in patients with severe COVID-19 infection," Médecine et Maladies Infectieuses, vol. 50, no. 4, p. 384, 2020.

[13] M. Mahévas, V.-T. Tran, M. Roumier et al., "Clinical efficacy of hydroxychloroquine in patients with covid-19 pneumonia who require oxygen: observational comparative study using routine care data," BMJ, vol. 369, p. m1844, 2020.

[14] E. S. Rosenberg, E. M. Dufort, T. Udo et al., "Association of treatment with hydroxychloroquine or azithromycin with inhospital mortality in patients with COVID-19 in New York state," JAMA, vol. 323, no. 24, pp. 1-10, 2020.

[15] P. Sarma, H. Kaur, M. Funk, and H. Kaur, "Virological and clinical cure in COVID-19 patients treated with hydroxychloroquine: A systematic review and meta-analysis," Journal of Medical Virology, vol. 92, no. 7, pp. 1-10, 2020.

[16] E. Chorin, M. Dai, E. Shulman et al., "The QT interval in patients with COVID-19 treated with hydroxychloroquine and azithromycin," Nature Medicine, vol. 26, no. 6, pp. 808-809, 2020.

[17] B. J. Drew, M. J. Ackerman, M. Funk et al., "Prevention of torsade de pointes in hospital settings," Circulation, vol. 121, no. 8, pp. 1047-1060, 2010.

[18] C. V. Etchegoyen, G. A. Keller, S. Mrad, S. Cheng, and G. Di Girolamo, "Drug-induced QT interval prolongation in the intensive care unit," Current Clinical Pharmacology, vol. 12, no. 4, pp. 210-222, 2018.

[19] A. Ramireddy, H. S. Chugh, K. Reinier et al., "Experience with hydroxychloroquine and azithromycin in the COVID-19 pandemic: Implications for QT interval monitoring," medRxiv, vol. 4, no. 22, 2020.

[20] W. Tang, Z. Cao, M. Han et al., "Hydroxychloroquine in patients with mainly mild to moderate coronavirus disease 2019: Open label, randomised controlled trial," BMJ, vol. 369, no. m1849, pp. 1-11, 2020.

[21] C. Jun, L. Ping, L. Li et al., "A preliminary study of hydroxychloroquine sulfate in the treatment of patients with common 2019 coronavirus disease (COVID-19)," Journal of Zhejiang University (Medical Sciences), vol. 49, no. 1, 2020.

[22] Z. Chen, J. Hu, Z. Zhang et al., "Efficacy of hydroxychloroquine in patients with COVID-19: Results of a randomized clinical trial," medRxiv, vol. 3, 2020.

[23] J. P. Higgins, J. J. Thomas, C. Al et al., Cochrane Handbook for Systematic Reviews of Interventions, pp. 117-188, John Wiley \& Sons, Hoboken, NJ, USA, 2nd edition, 2019.

[24] J. A. Sterne, M. A. Hernán, B. C. Reeves et al., "ROBINS-I: A tool for assessing risk of bias in non-randomised studies of interventions," BMJ, vol. 355, no. i4919, pp. 4-10, 2016.

[25] Modified newcastle - ottawa quality assessment scale, 2-3, http://Users/tamsulasoneT/Desktop/3/bjsports-2017December-51-23-1670-inline-supplementary-material-2.pdf.

[26] M. Saleh, J. Gabriels, D. Chang et al., "The effect of chloroquine, hydroxychloroquine and azithromycin on the corrected QT interval in patients with SARS-CoV-2 infection," Circulation, vol. 13, no. 6, 516 pages, 2020.

[27] E. Chorin, L. Wadhwani, S. D. M. Magnani et al., "QT interval prolongation and Torsade De Pointes in patients with COVID-19 treated with hydroxychloroquine/azithromycin," MedRxiv, vol. 19, no. 7, pp. 1425-1433, 2020.

[28] F. Bessière, H. Roccia, D. Antoine et al., "Assessment of QT intervals in a case series of patients with coronavirus disease 2019 (COVID-19) infection treated with hydroxychloroquine alone or in combination with azithromycininan intensive care unit," JAMA Cardiology, vol. 5, no. 9, p. 1067, 2020.

[29] P. Gautret, J. Lagier, P. Parola et al., "Clinical and microbiological effect of a combination of hydroxychloroquine and azithromycin in 80 COVID-19 patients with at least a six-day follow up: A pilot observational study," Travel Medicine and Infectious Disease, vol. 34, Article ID 101663, 7 pages, 2020. 
[30] B. Yu, C. Li, P. Chen, N. Zhou, and L. Wang, "Hydroxychloroquine application is associated with a decreased mortality in critically ill patients with COVID-19," MedRxiv, 2020.

[31] J. Magagnoli, S. Narendran, F. Pereira et al., "Outcomes of hydroxychloroquine usage in United States veterans hospitalized with Covid-19," Med, vol. 1, 1-14 pages, 2020.

[32] R. Barbosa Espe, R. Souza da Silva, F. Teiichi Costa Oikawa et al., "Empirical treatment with hydroxychloroquine and azithromycin for suspected cases of COVID-19 followed-up by telemedicine," 2020.

[33] N. Mercuro, C. Yen, D. J. Shim et al., "Risk of QT interval prolongation associated with use of hydroxychloroquine with or without concomitant azithromycin among hospitalized patients testing positive for coronavirus disease 2019," JAMA Cardiology, vol. 5, no. 9, pp. E1-6, 2020.

[34] A. Cipriani, A. Zorzi, D. Ceccato et al., "Arrhythmic profile and 24-hour QT interval variability in COVID-19 patients treated with hydroxychloroquine and azithromycin," International Journal of Cardiology, vol. 316, no. 20, pp. 280-284, 2020.

[35] T.-H. Yang, C.-Y. Chou, Y.-F. Yang, and Y.-P. Yang, "Systematic review and meta-analysis of the effectiveness and safety of hydroxychloroquine in COVID-19," Journal of the Chinese Medical Association, 2020.

[36] N. Mehta, M. Mazer-Amirshahi, N. Alkindi, and P. Ali, "Pharmacotherapy in COVID-19; A narrative review for emergency providers," American Journal of Emergency Medicine, vol. 2, pp. 1-6, 2020.

[37] A. Shamshirian, A. Hessami, and K. A. R. Heydari, "Hydroxychloroquine versus COVID-19: A periodic systematic review and meta-analysis hydroxychloroquine versus COVID-19: A periodic systematic review and meta-analysis," BMJ, 2020.

[38] A. Kumar, A. Singh, R. Singh, and A. Misra, "Hydroxychloroquine in patients with COVID-19: A systematic review," Diabetes \& Metabolic Syndrome: Clinical Research \& Reviews, vol. 14, no. 4, pp. 589-596, 2020.

[39] J. A. S. Jr, M. Bernaba, K. Yoshino, A. Ulhaque, and S. B. M. Kumar, "Systematic and statistical review of coronavirus disease 19 treatment trials," SN Comprehensive Clinical Medicine, vol. 2, pp. 1120-1131, 2020.

[40] H. Zhong, Y. Wang, Z.-L. Zhang et al., "Efficacy and safety of current therapeutic options for COVID-19 - lessons to be learnt from SARS and MERS epidemic: A systematic review and meta-analysis," Pharmacological Research, vol. 157, Article ID 104872, 2020.

[41] C. Jose and R. P. Gagan Bra, "Hydroxychloroquine in COVID-19: A systematic review and meta," BMJ, 2020.

[42] L. Ren, W. Xu, J. L. Overton, and S. Yu, "Assessment of hydroxychloroquine and chloroquine safety profiles-a systematic review and meta-analysis," MedRxiv, 2020.

[43] J. Sun, X. Deng, X. Chen et al., "Incidence of adverse drug reactions in COVID-19 patients in China: An active monitoring study by hospital pharmacovigilance system," Clinical Pharmacology \& Therapeutics, vol. 108, no. 4, p. 791, 2020.

[44] L. Jankelson, G. Karam, M. L. Becker, L. A. Chinitz, and M.-C. Tsai, "QT prolongation, torsades de pointes and sudden death with short courses of chloroquine or hydroxychloroquine as used in COVID-19: A systematic review," Heart Rhythm, vol. 17, no. 9, pp. 1472-1479, 2020.

[45] S. Gevers, M. S. G. Kwa, E. Wijnans, and C. van Nieuwkoop, "Safety considerations of chloroquine and hydroxychloroquine in treatment of COVID-19," European Society of
Clinical Microbiology and Infectious Diseases, vol. 26, no. 9, pp. 1276-1277, 2020.

[46] R. L. Mitra, S. A. Greenstein, and L. M. Epstein, "An algorithm for managing QT prolongation in coronavirus disease 2019 (COVID-19) patients treated with either chloroquine or hydroxychloroquine in conjunction with azithromycin: Possible benefits of intravenous lidocaine," HeartRhythm Case Reports, vol. 6, no. 5, pp. 244-248, 2020.

[47] D. Chang, M. Saleh, J. Gabriels et al., "Inpatient use of ambulatory telemetry monitors for COVID-19 patients treated with hydroxychloroquine and/or azithromycin," American College of Cardiology Foundation, vol. 75, no. 23, pp. 2992-2993, 2020.

[48] M. A. Chary, A. F. Barbuto, S. Izadmehr, B. D. Hayes, and M. M. Burns, "COVID-19: therapeutics and their toxicities," Journal of Medical Toxicology, vol. 16, no. 3, pp. 1-11, 2020. 